\title{
Experiments on a smooth wall hypersonic boundary layer at Mach 6
}

\author{
Dominik Neeb $^{1}$ (D) Dominik Saile ${ }^{1} \cdot$ Ali Gülhan ${ }^{1}$
}

Received: 21 August 2017 / Revised: 3 February 2018 / Accepted: 15 February 2018 / Published online: 22 March 2018

(c) The Author(s) 2018

\begin{abstract}
The turbulent boundary layer along the surface of high-speed vehicles drives shear stress and heat flux. Although essential to the vehicle design, the understanding of compressible turbulent boundary layers at high Mach numbers is limited due to the lack of available data. This is particularly true if the surface is rough, which is typically the case for all technical surfaces. To validate a methodological approach, as initial step, smooth wall experiments were performed. A hypersonic turbulent boundary layer at $M a=6\left(M a_{e}=5.4\right)$ along a $7^{\circ}$ sharp cone model at low Reynolds numbers $\operatorname{Re}_{\theta} \approx 3000$ was characterized. The mean velocities in the boundary layer were acquired by means of Pitot pressure and particle image velocimetry (PIV) measurements. Furthermore, the PIV data were used to extract turbulent intensities along the profile. The mean velocities in the boundary layer agree with numerical data, independent of the measurement technique. Based on the profile data, three different approaches to extract the skin friction velocity were applied and show favorable comparison to literature and numerical data. The extracted values were used for inner and outer scaling of the van Driest transformed velocity profiles which are in good agreement to incompressible theoretical data. Morkovin scaled turbulent intensities show ambiguous results compared to literature data which may be influenced by inflow turbulence level, particle lag and other measurement uncertainties.
\end{abstract}

\section{Introduction}

The thin flow region close to the surface of high-speed vehicles, dominated by the boundary layer, drives shear stress and heat flux. Therefore this region essentially influences the vehicle design, including components like aerothermal protection systems. This is in particular true if the boundary layer is turbulent, which is connected to increased gradients in the flow and therefore increased shear and heat flux. The understanding of compressible turbulent boundary layers at high Mach numbers is limited due to the lack of available data. This is especially true if additional aspects like surface roughness influence the flow. For the spacecraft design, this typically results in large safety margins which mean an increase in weight and cost. Therefore, further experimental data are important for the physical understanding and validation of state-of-the-art computations. However, accurate measurements of the compressible turbulent boundary layer in ground-based facilities is very challenging due

Dominik Neeb

dominik.neeb@dlr.de

1 Institute of Aerodynamics and Flow Technology, Supersonic and Hypersonic Technologies Department, German

Aerospace Center (DLR), Cologne, Germany to a multitude of reasons, e.g., limited model dimensions, thin boundary layers, limited useable test times and inflow effects, like turbulence intensity. The choice of measurement technique is also a crucial parameter with high impact on spatial and temporal resolution.

At high speeds, density gradients influence the properties of the compressible boundary layer. Viscous heating within the boundary layer, which is caused by the deceleration of the fluid, is responsible for high-density gradients. But also the wall temperature affects the near wall density. This complicates the definition of driving parameters for the flow, like the Reynolds number. For incompressible flow, typically quantities of the free-stream or boundary layer edge conditions like density $\rho_{e}$, velocity $u_{e}$ and viscosity $\mu_{e}$ are combined with a characteristic boundary layer length scale, such as the momentum loss thickness $\theta$, to $\operatorname{Re}_{\theta}=\rho_{e} u_{e} \theta / \mu_{e}$. Due to the changing density in the compressible boundary layer, this definition might not be sufficient to capture, e.g., wall effects. This led to the definition of $\operatorname{Re}_{\delta 2}=\rho_{e} u_{e} \theta / \mu_{w}$, where the viscosity is defined at the wall, instead of the boundary layer edge, according to, e.g., Fernholz and Finley (1980), Duan and Martin (2011), Duan et al. (2011).

One attempt to study compressible boundary layers is to enable comparison to the larger database of the incompressible counterpart. This is done by accounting for the density 
gradient with specific scalings or transformations. A prominent and widely applied transformation for the mean velocity is the van Driest scaling (Van Driest 1951, 1956). It shows good results in a broad Mach number range at a variety of experimental (e.g., Humble et al. 2007; Ekoto et al. 2008; Sahoo et al. 2009; Williams 2014) and numerical data (e.g., Duan et al. 2010, 2011). Due to the thin boundary layer and the complexities in resolving this region accurately in experimental measurements, data is typically limited to regions excluding the region close to the wall. Humble et al. (2007) reported to be the first to perform PIV measurements within the viscous sublayer of a supersonic boundary layer at $M a=2.1$.

A further transformation was postulated by Morkovin with his hypothesis that the turbulent intensities within the boundary layer also scale with the mean density profile and skin friction (Morkovin 1962). It was based on the suggestion that the length scales are not significantly affected by compressibility and that turbulent coherent structures should follow an incompressible pattern. Morkovin demonstrated his scaling with data up to Mach numbers of 4.76. Only a few investigations exist on the validity for higher Mach numbers, like the PIV measurements at $M a_{e} \approx 7$ according to Williams (2014), Williams et al. (2015), Williams and Smits (2017) and Williams et al. (2018) or data from DNS simulations, e.g., Duan et al. (2011) and Priebe and Martin (2011).

At the German Aerospace Center (DLR) Cologne, an experimental setup was characterized in the hypersonic wind tunnel, with the objective of realizing accurate boundary layer measurements on smooth and rough wind tunnel models. Before applying the experimental setup on rough wall models, a series of test campaigns was conducted to characterize the performance with measurements on a sharp smooth $7^{\circ}$ cone model, based on literature and CFD data. This paper summarizes the results of these smooth wall characterization tests. The main focus lay on the measurement of the mean velocity profiles in the logarithmic part of the boundary layer. This is of special interest for future rough wall investigations, since specific roughness characterizing parameters can be deduced from the velocity defect in this region (see, e.g., Bowersox 2007). Two different independent measurement techniques were applied, first with a movable Pitot probe and second with particle image velocimetry (PIV). Dedicated uncertainty analyses were performed for both measurement techniques. For PIV measurements, influences like the impact of particle slip on the mean velocity and turbulent quantities were approximated based on numerical predictions. The mean flow velocities from both measurement techniques were used for a cross-check and for different analyses according to AGARD suggestions (Fernholz and Finley 1980). For these analyses, van Driest and skin friction velocity scaling were necessary. Since no direct measurement of the skin friction velocity, defined by
$U_{\tau}=\sqrt{\tau_{w} / \rho_{w}}$, with the wall shear stress $\tau_{w}$ and wall density $\rho_{w}$, was performed, different indirect approaches were applied and compared to each other and literature data. Additionally, turbulent intensity profiles were extracted from velocity data of the PIV measurements. Limitations in number of images and resolution as well as random noise and sub-grid filtering at the demanding flow conditions complicated the analysis of the data and is discussed. Morkovin's scaling was applied on the final results and compared to literature data.

In the following Sect. 2, analytical and numerical tools are presented, which were used to support the experimental analysis. The applied experimental tools and procedures are summarized in Sect. 3. Before presenting the results, dedicated sensitivity studies and uncertainties are discussed for the numerical and experimental data in Sect. 4. The results for the hypersonic boundary layer mean flow velocities and turbulence intensities are then presented and discussed in Sect. 4. Finally, conclusions are given in Sect. 5. If not otherwise stated, the axial, wall-parallel and wall-normal directions are denoted in the following by $x, s$ and $y$, respectively. Velocities denoted by $U$ correspond to the $s$ and $V$ to the $y$ direction. Furthermore, mean velocities are denoted by a capital letter $(U, V)$, while instantaneous velocities are the corresponding lowercase letters $(u, v)$. The fluctuating velocities are denoted by an additional bar $\left(u^{\prime}, v^{\prime}\right)$. An overbar indicates ensemble averaging (e.g., $\overline{u^{\prime} u^{\prime}}$ ). Superscript + indicates normalization using viscous length and velocity scales which are $v_{w} / U_{\tau}$ and $U_{\tau}$, respectively, where $v_{w}$ is the kinematic viscosity at the wall.

\section{Analytical and numerical tools}

In this chapter, analytical and numerical tools are summarized, which were used to support the analysis of experimental data. First, the analytical predictions for the skin friction are listed. In the following, the hybrid CFD code TAU will be introduced for the rebuilding of skin friction, boundary layer mean velocity and turbulence intensity profiles.

\subsection{Analytical prediction methods}

In the framework of this paper, a correlation based on the work of Van Driest for turbulent compressible flow along a flat plate with heat transfer using Sutherland's viscosity model (extended for low temperatures according to Abgrall et al. 1992) was used according to Van Driest (1951) and Willems and Gülhan (2013). A transformation from a flat plate to a cone skin friction value according to Van Driest (1952) was used in this paper. 


\subsection{TAU code}

The CFD calculations were performed with the DLR TAU code. The TAU code is a finite volume Euler/ Navier-Stokes solver, which can use structured, unstructured and hybrid meshes, and has already been applied and validated on studies of various configurations in various flow regimes, including hypersonic flow (Hannemann 2002). In case of turbulent computations, a one-equation Spalart-Allmaras model with Edwards modification (SAE), a two-equation Wilcox- $k-\omega$ model and a sevenequation Reynolds stress model (RSM) with a hybrid Speziale-Sarkar-Gatski/Launder-Reece-Rodi (SSG/LRR- $\omega$ ) model was used. If not otherwise noted, a turbulent Prandtl number of $P r_{t}=0.9$ was assumed in all applied models. This choice is supported by DNS data according to Duan et al. (2010). A dedicated grid convergence anaylsis was performed (Sect. 4.2).

\subsubsection{Particle module extensions}

An existing analysis module for the TAU solver, based on a Lagrangian approach, was used to model motions of particles with mass. This module was originally implemented to predict ice accretion on various components of an aircraft, by predicting droplet trajectories in CFD solutions (Widhalm et al. 2008). Since this module is designed for sub- to transonic flows, it was necessary to extend the implementation of the equations of motion to cover compressible, rarefied conditions. This included implementation of suitable drag coefficient models, chosen with the Tedeschi (Tedeschi et al. 1999) and Henderson model (implemented as given in Thomas 1991). Both include the effect of compressibility and variable Knudsen number. The TAU module was validated with literature data from Tedeschi et al. (1999). With the presented module, single particle trajectories, as well as wall particle impingement points and particle density distributions along the conical flow field, can be modeled, extracted and compared to experimental data. Analyses with this approach are presented in Sect. 4.3.

\section{Experimental tools and procedures}

In this section, the different measurement techniques are presented. First, details of the pressure measurements are given. Then, the setup and analysis tools of the particle image velocimetry (PIV) are reported. Finally, different methods to approximate the skin friction velocity from mean velocity profiles are presented.

\subsection{Wind tunnel and model properties}

Experiments were performed in the DLR hypersonic wind tunnel $(\mathrm{H} 2 \mathrm{~K})$ in Cologne. The facility is an intermittently working blowdown tunnel with a free jet test section. Depending on the flow condition, test durations up to $30 \mathrm{~s}$ can be achieved. The facility can be equipped with five exchangeable contoured nozzles with an exit diameter of $600 \mathrm{~mm}$ for different Mach numbers, i.e., $M a=5.3 ; 6.0 ; 7.0 ; 8.7 ; 11.2$. To avoid air condensation as well as to operate the facility at high stagnation temperatures, electrical heaters with a capacity of up to $5 \mathrm{MW}$ are integrated upstream of the nozzle. Unit Reynolds numbers between $R e=2.5 \times 10^{6}$ to $16 \times 10^{6} \mathrm{~m}^{-1}$ can be set by varying the stagnation pressure $p_{0}$ and stagnation temperature $T_{0}$.

To enable natural transition from a laminar to turbulent boundary layer, without the necessity of a tripping device along the model, a sharp cone with $7^{\circ}$ opening angle was chosen. The model consisted of three exchangeable segments. The first segment consisted of a sharp metallic nose with a radius in the order of $0.1 \mathrm{~mm}$. All other segments were made of polyether ether ketone (PEEK), which is a colorless organic high-temperature polymer thermoplastic. It is a standard material for heat flux investigations in $\mathrm{H} 2 \mathrm{~K}$ due to detailed knowledge of the temperature-dependent material properties (Häberle 2009). The total length of the model was $L=0.73 \mathrm{~m}$ with an end diameter of $0.18 \mathrm{~m}$. The model was fixated by a typical sting configuration and placed in a short distance downstream of the wind tunnel nozzle exit (see Fig. 1).

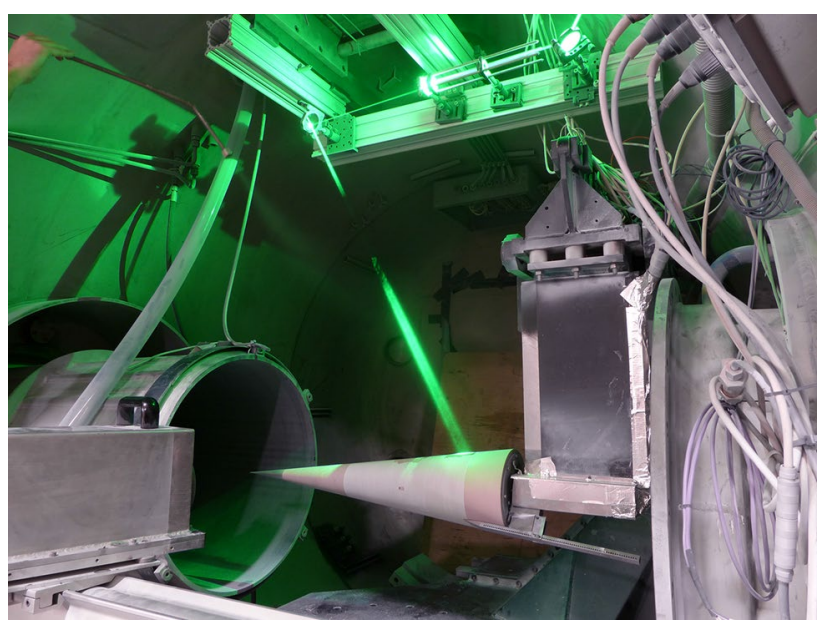

Fig. 1 PIV setup in $\mathrm{H} 2 \mathrm{~K}$ 


\subsection{Pressure measurements}

\subsubsection{Surface pressure}

The wind tunnel model was equipped with pressure holes with a diameter of $0.5 \mathrm{~mm}$ at nine different positions. Pressure tabs were located at three different axial sections along the model, at $x=0.25,0.495$ and $0.651 \mathrm{~m}$. In the first and last section, four different tabs were positioned with an angular distance of $45^{\circ}$. This setup enabled the analysis of model alignment and pressure gradients along the surface. The tabs were connected via steel and flexible tubing to a miniature electronic pressure scanner (ESP) outside the wind tunnel model with a measurement range of $34.5 \mathrm{kPa}(5 \mathrm{psi})$.

\subsubsection{Pitot pressure}

The Pitot pressure was measured with a movable rake, consisting of five tubes, each with an inner and outer diameter of 0.2 and $0.4 \mathrm{~mm}$, respectively. The distance between subsequent tubes was $1 \mathrm{~mm}$. This multiple tube concept minimized the testing time for a highly resolved boundary layer profile. In wall-normal direction the rake was traversed with a linear actuator $L 4118 L 1804-T 6 X 1$ from Nanotec Electornic $\mathrm{GmbH} \& \mathrm{Co}$. KG, whereas the axial position was fixed during a test run. The motor was operated by a Nanotec SMCI33-1 controller. The position of the probe was monitored with a Nanotec WEDL 5546 encoder. The actuator has a minimal step resolution of $5 \mu \mathrm{m}$ and a maximum speed of $20 \mathrm{~mm} / \mathrm{s}$. The rake movement during test time was stepwise with corresponding settling times to ensure a sufficient filling of the tube volumes. The Pitot tubes were connected via steel and flexible tubing to an ESP scanner with a measurement range of $103.4 \mathrm{kPa}(15 \mathrm{psi})$. The impact of potential interaction problems between the multiple tubes of the rake was tested with measurements via a single tube reference device. The differences fell within the corresponding uncertainties. To account for small run-to-run inflow differences, the pressure data of each run were first transformed to pressure coefficients via $c_{p}=\left(p_{\text {Pitot }}-p_{\infty}\right) / q_{\infty}$. With the nominal inflow values of static pressure $p_{\infty}$ and stagnation pressure $q_{\infty}$ according to Table 1, a corresponding Pitot pressure $p_{\text {Pitot }}$ was calculated and combined to a complete profile from several runs. The data from surface and Pitot pressure measurements were transformed to velocity profiles applying the
Rayleigh Pitot equation (see, e.g., Staff 1953) and assuming a Crocco-Busemann temperature distribution within the boundary layer (see, e.g., White 2006). The selection of the temperature distribution was based on a comparison between different approximations and numerical profiles for the chosen $\mathrm{H} 2 \mathrm{~K}$ flow conditions.

\subsection{Particle image velocimetry}

\subsubsection{Setup}

A particle image velocimetry setup was used to measure two-dimensional, two-component flow velocity fields. An ULTRA CFR Nd:YAG Laser System of the company Quantel was used as light source. It consisted of two pulse lasers which were superimposed and converted to a wavelength of $\pm 532 \mathrm{~nm}$. The frequency was set to $15 \mathrm{~Hz}$ for a maximum energy of $191 \mathrm{~mJ}$ per pulse. The final laser sheet along the model surface had a nominal dimension of $60 \times(0.5-1.0) \mathrm{mm}$ (streamwise length $\times$ thickness). The setup inside the test section is visible in Fig. 1. To reduce wall reflections, the PEEK model wall was locally painted with red color in the vicinity of the laser sheet impact region. Additionally, the sheet was positioned so that most part of its extent was located on the camera-averted side of the model. For the acquisition of images, a PCO 1600 camera system was used, with a CCD chip of maximum resolution of $1600 \times 1200 \mathrm{pix}^{2}$. The camera was positioned outside of the test section, at a distance of approximately $2.1 \mathrm{~m}$ from the model. A K2 DistaMax long distance microscope from the company Infinity Photo Optical was used. The chosen setup resulted in a magnification of $M \approx 0.99$. An optical filter (wavelength $532 \pm 2 \mathrm{~nm}$ ) was mounted between the objective and the CCD chip. With this setup a region of interest (ROI) of $9.0 \times 12.0 \mathrm{~mm}^{2}$ (streamwise $\times$ wall-normal distance) was obtained. The location of this ROI is described in Sect. 4. For seeding, a solid particle generator (SPG) was used, which was connected to the stilling chamber of the $\mathrm{H} 2 \mathrm{~K}$ wind tunnel. $\mathrm{TiO}_{2}$ particles of type 1002 from the company KRONOS INTERNATIONAL INC. were used. The manufacturer states a median diameter of $d_{p} \approx 0.2-0.3 \mu \mathrm{m}$ and a density of $\rho_{p} \approx 3800 \mathrm{~kg} / \mathrm{m}^{3}$. Before seeding, the particles were sieved and than dehydrated in an oven at $70^{\circ} \mathrm{C}$ for $t>6 \mathrm{~h}$ to $12 \mathrm{~h}$ to prevent agglomeration (see, e.g., Ragni et al. 2011). A dedicated sensitivity study of the particle
Table 1 Nominal flow conditions FC1 and FC2

\begin{tabular}{lllllll}
\hline Flow cond. & State & $M a(-)$ & $R e\left(10^{6} 1 / \mathrm{m}\right)$ & $p_{0}(\mathrm{bar})$ & $T_{0}(\mathrm{~K})$ & $U(\mathrm{~m} / \mathrm{s})$ \\
\hline FC1 & Inflow & 6.06 & 15.7 & 20.0 & 500 & 940 \\
& Edge & 5.40 & 20.5 & 19.8 & 500 & 926 \\
FC2 & Inflow & 6.06 & 16.0 & 18.9 & 475 & 916 \\
& Edge & 5.40 & 21.0 & 18.7 & 475 & 903 \\
\hline
\end{tabular}


property impact on the response behavior is given in Sect. 4. The timing of the PIV system was controlled via a LabSmith LC880 8-channel trigger box. The interframing time between two images was set to nominally $t_{p}=120 \mathrm{~ns}$. During testing, a dependence of measured velocities on the laser power level was noticed. The time difference between two subsequent laser pulses was identified as the main contributor to this deviation. A dedicated laser characterization with a photodiode setup was performed after the test campaign. This characterization resulted in corrected pulse distance values for all preset pulse distance and power level combinations used during the test campaign. The standard uncertainty of the extracted deviations was in the order of $1 \mathrm{~ns}$ or below and were included in the total PIV measurement uncertainty (see Sect. 4). A comparable influence was also reported in Williams (2014). The described setup typically resulted in a mean freestream displacement of $\Delta x \approx 14.5$ pix.

\subsubsection{Analysis}

All analyses were performed with the commercial PIVview suite version 3.6.0. Approx. 200 valid PIV images were recorded per run. Several image pre-processing steps were conducted before analysis. Due to small model vibrations, a pattern matching algorithm was used to normalize all recorded images first. The wall was identified based on intensity images, averaged over all images per run. Profiles in the wall-normal direction, accumulated in streamwise direction, were used. A small rotation, typically below approximately $1^{\circ}$, was applied to align the surface with the horizontal level. A background image subtraction was omitted since no significant improvement was noticeable.

For the processing of the images, several validation criteria were defined and correlated velocity vectors were not accepted if one of the following three criteria were not met. First, a normalized median filter with a threshold of 8 was applied on each vector along with its eight neighbors. Second, a dynamic mean test with a mean value of 2.0 and variance of 1.0 was applied. The third criterion was a minimum signal-to-noise ratio of each correlation peak of 5.0. An interrogation window size was accepted only if at each vector position at least $90 \%$ of the corresponding images met the above criteria. The remaining single image non-accepted vectors were not included in further analysis (no interpolation was used). Based on these criteria, several single-grid and multigrid interrogation windows were chosen for further analyses (see Sect. 4). If not otherwise noted, an overlap of $50 \%$ was used. In all cases an algorithm was applied with standard FFT correlation using Whittaker reconstruction sub-pixel peak fitting. Additionally, the algorithm used a multiple-pass interrogation method, which applied image deformation via cubic B-spline interpolation on all 3 selected steps, to compensate for high shear near the wall. Ensemble correlation analysis was applied to selected data.

\subsection{Methods for skin friction approximation}

For the analysis of the boundary layer velocity profiles, inner scaling is of interest (see Sect. 4). Since no direct measurements of the wall skin friction were performed, several different indirect approaches were compared. First, a fitting procedure, based on the law-of-the-wall, is presented. Additionally, the velocity gradient from the wall closest data points of the PIV measurements was exploited. Besides that, a modified integral method to approximate the compressible wall skin friction along the cone model was applied.

Fitting approach A procedure was applied to fit a measured mean velocity profile onto the law-of-the-wall. In addition to the logarithmic part, the wake part was also included using a Coles wake parameter formulation. The law-of-thewall for a compressible turbulent boundary layer can be given according to White (2006):

$U_{\mathrm{eff}}^{+}=\frac{U_{\mathrm{eff}}}{U_{\tau}}=\frac{1}{\kappa} \ln \left(y^{+}\right)+B+\frac{\Pi}{\kappa} 2 \sin ^{2}\left(\frac{\pi}{2} \frac{y}{\delta}\right)$.

Included are the effective velocity $U_{\text {eff }}$, derived from the absolute, compressible velocity $U$ by applying the transformation after Van Driest (see, e.g., Van Driest 1951, 1956; Berg 1977), the normalized wall normal distance $y^{+}=\sqrt{y \cdot U_{\tau} / \nu_{w}}, B$ as the law-of-the-wall constant chosen with $B=5.0, \kappa$ as Karman constant chosen with $\kappa=0.4, \Pi$ as the wake strength parameter, usually depending on inflow and pressure gradient, and $\delta$ as the boundary layer thickness (defined here by the wall distance where the velocity has reached $99 \%$ of the edge velocity). Including the wake formulation increased the amount of useful data points within the boundary layer, which in turn enhanced the quality of the fitting procedure. The applied method was originally introduced to study turbulent boundary layer profiles along smooth and rough walls by Berg (1977). The parameters necessary to fit are the skin friction velocity $U_{\tau}$ and the Coles wake parameter $\Pi$. This method and its algorithm were validated against the data of Berg. Several consistency tests were performed by applying the fitting approach on PIV results derived with different interrogation windows (reported in Sect. 4.3) and on results from ensemble correlation. These tests resulted in a percentage change of the derived skin friction velocities of $\Delta U_{\tau}<1.3 \%$, which showed only a small sensitivity of the analysis to the investigated parameters. Additionally, it is a sign for the robustness of the fitting procedure, since the results are only slightly affected by the amount of points within the profile, especially in the logarithmic region. The insensitivity of the fitting approach to the number of points is especially helpful for the analysis 
of the Pitot data, which had a lower resolution than the PIV data.

Gradient approach If the velocity profile in the viscous sublayer close to the wall is available, the wall shear stress may be approximated by the velocity gradient at the wall. The high wall-normal resolution of the PIV interrogation windows $8 \times 512$ pix $^{2}$ profiles (given as wall-normal $\times$ streamwise dimensions in pixel) enabled a distance from the wall for the first data point of $i y(1)=4$ pix which corresponds to $y(1)=0.03 \mathrm{~mm}, y / \delta(1)=0.0087, y^{+}(1)=1.79$ which is located well within the viscous sublayer (see Fig. 5). Due to the derivation of the van Driest velocity transformation, its applicability is limited to the logarithmic layer (e.g., Fernholz and Finley (1980), Williams (2014)). For the viscous sublayer, an alternative velocity transformation can be derived from the shear stress budget in the sublayer with the assumption of negligible turbulent stress contribution, an exponential viscosity law with the exponent $\omega=1$, the Crocco temperature distribution, $\partial p / \partial x=0$ and constant wall temperature $T_{w}$ according to Fernholz and Finley (1980). For the PIV data in this paper, both transformations showed only minor differences in the sublayer, which fell below the corresponding uncertainties (see Sect. 4.3). Different developments to derive a scaling which is applicable to both, the viscous sublayer and log-layer, were discussed, e.g., by Patel et al. (2016) and Trettel and Larsson (2016). Trettel and Larsson (2016) derived a scaling tested on supersonic channel and boundary layer cases with nonadiabatic wall conditions, including the effect of wall heating. To categorize the impact of wall heating the authors derived a dimensionless parameter with $B_{q}=q_{w} /\left(c_{p} u_{\tau} T_{w}\right)$, where $q_{w}$ represents the wall heat flux (negative, if wall is heated) and $c_{p}$ the heat capacity at constant pressure. Low absolute values of $B_{q}$ represent near adiabatic conditions. For boundary layer cases with $\left|B_{q}\right|<0.069$, only small differences between the introduced and the classical van Driest scaling were visible (see Trettel and Larsson 2016). For the boundary layer discussed in this paper, a value of $\left|B_{q}\right| \approx 0.02$ can be approximated, based on infrared thermography data. This represents a case with only limited heating of the wall $\left(T_{w} / T_{a w} \approx 0.8\right)$ and therefore only limited differences to the van Driest scaling are expected. Therefore, the van Driest scaled velocities were used in all following analyses. To estimate the shear stress, the wall closest velocity points were used to estimate the corresponding partial derivative by a difference quotient. The necessary wall viscosity $\mu_{w}$ was calculated via the Sutherland law based on the corresponding wall temperature from infrared thermography. The velocity difference was calculated for all points within $y^{+}<15$, assuming zero velocity at the wall.

Integral approach This method is based on an approach originally implemented to approximate the shear srtress along a rough flat plate wall according to Latin (1998). For this approach, the momentum integral equation is solved in between two consecutive positions along the surface by implementing a functional dependence for the skin friction coefficient of the kind $c_{f, e}=f(\theta)$ (formulated with the boundary layer edge conditions). This function can be derived with the law of the wall solved at the boundary layer edge. In case of the rough version of the law of the wall along a flat plate, the resulting momentum integral can be solved analytically (Latin 1998). In case of a compressible smooth cone flow, an analytical solution is not possible anymore and need to be solved numerically. For the method, the validity of the law-of-the-wall needs to be assumed. Formulated at the boundary layer edge, Eq. 1 may be inserted into the compressible zero-pressure gradient momentum integral equation, which can be formulated along a cone with the assumption of constant edge conditions according to Fenter (1960). The resulting equation may be solved numerically with a Runge-Kutta solver with known initial values for boundary layer running length and momentum thickness. In this paper, the values were extracted from the first PIV (or Pitot) measurement section. The equations were iterated so that the resulting $\theta_{2}$ matches the data at the second PIV (or Pitot) measurement section at $x_{2}$. The influence of the numerical step size $\Delta x$ was tested and found to be negligible for the conditions in this paper. The procedure was successfully cross-checked with the original implementation of Latin (1998) in a flat plate implementation. Additionally, the procedure was tested and validated with numerical data along a cone. The resulting percentage deviations of the skin friction coefficient was typically $\left|\Delta c_{f, e}\right| \leq 1.2 \%$.

\section{Results and discussion}

Section 4.1 contains the nominal flow conditions of the conducted experimental test campaigns and the definition of measurement sections to extract boundary layer data. Then, numerical sensitivity studies are reported in Sect. 4.2. After the discussion of uncertainties and sensitivities, the experimental results of the mean velocities are compared to numerical and literature data in Sect. 4.3. Finally, the analysis of turbulence intensities from PIV measurements is discussed based on numerical and literature data in Sect. 4.4.

\subsection{Nominal flow conditions}

Table 1 contains the nominal flow conditions of the wind tunnel campaigns. Two different flow conditions FC1 and FC2 were defined and details about the Mach number $M a$, unit Reynolds number $R e$, reservoir pressure $p_{0}$, reservoir temperature $T_{0}$ and velocity $U$ are given. The values are reported for the inflow and boundary layer edge condition. 
In case of the inflow state $(\infty), U$ denotes the axial velocity, in contrast to the wall-parallel component in the rest of the paper. The inflow Mach number is derived from nozzle calibration, including viscous effects, depending on the Reynolds number. The reservoir conditions were directly measured, whereas the static conditions of the inflow were calculated via gas dynamic equations (e.g., Staff 1953). The uncertainties of the inflow parameters are based on calibrations of the corresponding instrumentation and can be given for the reservoir pressure with $\pm 0.1 \%$ (full scale of $p_{0}=70$ bar $)$, reservoir temperature with $\pm 1.1^{\circ} \mathrm{C}$ or $\pm 0.4 \%$ (whichever is higher) and inflow Mach number with \pm 0.04 . The nominal edge conditions were derived by the Taylor-Maccoll equation (see, e.g., Anderson 1990), assuming negligible influence of a thin boundary layer. The two slightly different inflow conditions were designed to match the Mach and Reynolds number for different reservoir conditions. This was necessary due to infrastructure refurbishments of the $\mathrm{H} 2 \mathrm{~K}$ facility. The PIV measurement campaign was performed with FC1, and the Pitot campaign with FC2.

Table 2 contains detailed information for every reported run in this paper. The reservoir conditions of the conducted runs typically differ by less than approximately $1 \%$ from the nominal values. Run numbers from 7 to 10 correspond to the PIV campaign at FC1 and from 13 to 52 to the Pitot campaign at FC2. Only runs with sufficient quality with respect to inflow conditions and boundary layer measurements were chosen and included in this paper. The table contains the actual Reynolds number $R e_{\infty}$ ( $M a_{\infty}=6.06$ for all runs), static pressure $p_{\infty}$, temperature $T_{\infty}$ and velocity $U_{\infty}$. Additionally, the streamwise location for boundary layer profile measurements is given with measurement section MS1 or MS2 besides corresponding measurement techniques (MT). Locations for both measurement sections and measurement techniques are defined in Table 3 with the axial and the surface running length $x$ and $s$, respectively.
Table 3 Streamwise locations of profile measurement sections for Pitot and PIV measurements

\begin{tabular}{llllll}
\hline Name & Pitot & & & PIV \\
\cline { 2 - 3 } \cline { 5 - 6 } & $x(\mathrm{~mm})$ & $s(\mathrm{~mm})$ & & $x(\mathrm{~mm})$ & $s(\mathrm{~mm})$ \\
\hline MS1 & 492.3 & 496.0 & & $490.3-499.3$ & $493.9-503.0$ \\
MS2 & 630.2 & 635.0 & & $623.8-632.8$ & $628.5-637.6$ \\
\hline
\end{tabular}

\subsection{Numerical sensitivity studies}

Dedicated sensitivity studies were performed on the hybrid axisymmetric mesh of the $7^{\circ}$ cone model. The spatial convergence was checked on three grid refinement levels via grid convergence index (GCI) according to Slater et al. (2000) and Roache (1994). The necessary parameters for a study with three grids ( $i=1$ : fine, $i, j=2$ : medium, $i, j=3$ : coarse) were used according to Slater et al. (2000). All TAU code grid convergence computations were performed with the RSM model for fully turbulent flow and the nominal input parameters for FC1 and $T_{w}=340 \mathrm{~K}$ (see Table 1). A nearly constant refinement ratio of $r \approx 1.5$ was realized by globally changing a pre-defined grid resolution in the complete field downstream the shock region. Additionally, the number of structured sub-grid layers along the wall was refined by an equal ratio (fine: 60 layers, medium: 40 layers, coarse: 27 layers). The corresponding order of convergence was calculated to $p=1.80$, which is close to the value $p=1.75$, suggested by Rakowitz (2002) for TAU calculations with mixed first and second-order methods. The solution value to extract the GCI was the wall heat flux at MS1. The resulting convergence index between the finest and medium mesh resulted in $G C I_{12}=2.08 \%$. The ratio $G C I_{23} /\left(r^{p} G C I_{12}\right)=1.02$ showed that the grid solutions were in the asymptotic range. Besides spatial convergence, the heat flux evaluation scheme, the height of first grid layer above the wall, reconstruction of gradients and turbulence model were considered. All influences were finally added in quadrature and resulted in a total

Table 2 Test matrix

\begin{tabular}{lllllll}
\hline Run no. & $R e_{\infty}\left(10^{6} 1 / \mathrm{m}\right)$ & $p_{\infty}(\mathrm{bar})$ & $T_{\infty}(\mathrm{K})$ & $U_{\infty}(\mathrm{m} / \mathrm{s})$ & MS $(-)$ & MT $(-)$ \\
\hline 7 & 15.7 & 0.0120 & 60 & 941 & 1 & PIV \\
8 & 15.8 & 0.0119 & 59 & 937 & 1 & PIV \\
10 & 15.6 & 0.0119 & 60 & 942 & 2 & PIV \\
13 & 15.9 & 0.0116 & 58 & 927 & 2 & Pitot \\
23 & 16.0 & 0.0113 & 57 & 917 & 2 & Pitot \\
24 & 16.0 & 0.0113 & 57 & 918 & 2 & Pitot \\
25 & 16.0 & 0.0111 & 56 & 913 & 2 & Pitot \\
32 & 16.0 & 0.0113 & 57 & 917 & 1 & Pitot \\
33 & 15.8 & 0.0112 & 57 & 918 & 1 & Pitot \\
39 & 16.0 & 0.0113 & 57 & 916 & 1 & Pitot \\
52 & 15.9 & 0.0112 & 57 & 916 & 1 & Pitot \\
\hline
\end{tabular}


variation of $\Delta \dot{q} \approx 13.2 \%, \Delta c_{f} \approx 9.2 \%\left(\Delta U_{\tau} \approx 4.6 \%\right)$ and $\Delta p_{w} \approx 0.5 \%$, which need to be accepted for TAU results on the finest grid. The turbulence model variation had the biggest impact with $\Delta \dot{q} \approx 10-13 \%$. If not otherwise stated, all results for comparison to experimental data were performed on the finest grid.

\subsection{Mean flow velocity data}

This chapter contains the results of the mean flow velocities in the cone boundary layer extracted via Pitot pressure and PIV measurements. First, consistency, sensitivity and uncertainty checks of the PIV data are reported. Afterward, a synthesis for the profiles from both measurement techniques with numerical and literature data is given. Then, different approaches to extract the skin friction velocity from the Pitot and PIV data are presented and characterized, based on analytical and numerical data. Finally, the selected profiles are analyzed in inner and outer scaled form and discussed based on literature data.

\subsubsection{Uncertainties and sensitivities}

For the PIV data, general uncertainties from the setup, sensitivities to interrogation windows, wall temperature, particle slip and image number convergence were investigated. If not otherwise noted in this paper, combined uncertainties were derived by linear propagation of uncertainties. This uncertainty value represents a maximum level considering the most unfavorable and improbable case when all independent influencing variables reach their minimum or maximum uncertainty value.

Setup The uncertainties for the flow velocities derived by PIV was estimated to $\pm 2.5 \%$ based on the edge velocity. The given final percentage difference is a propagated result of uncertainties for typical setup uncertainty, lens distortion, laser pulse timing and calibration reading error.

Interrogation window Sensitivity studies were performed with synthetic PIV images based on TAU RSM data of the cone flow to test the influence of the interrogation window (iw) size on the mean velocity profiles. Especially, the impact of stretched, high aspect ratio, interrogation windows was of interest. This type of window was selected to increase the wall-normal resolution, but at the same time ensure an acceptable amount of particles per interrogation window. This window shape is supported by the nature of the boundary layer with a much higher streamwise than wall-normal velocity magnitude. Interrogation window sizes were selected according to the analysis of the experimental data with $64 \times 512$ pix $^{2}, 32 \times 512$ pix $^{2}, 16 \times 512$ pix $^{2}$ and $8 \times 512$ pix $^{2}$ and multigrid analysis with $256 \times 256$ to $64 \times 96$ pix $^{2}$ (published in Neeb et al. 2015) (given as wallnormal $\times$ streamwise dimensions in pixel). Valid results could be extracted from the synthetic images for all tested interrogation windows. Only in the immediate vicinity of the wall $(y / \delta \approx 0.1)$, differences of pixel shift above $\Delta d x=0.1$ pix (corresponding to $0.7 \%$ of the edge velocity) were detected which were mainly caused by increased velocity gradients in this region. The drawback of using the aforementioned high aspect ratio interrogation windows is a limited resolution in the streamwise direction. Estimations based on TAU simulations resulted in an uncertainty below $\Delta U / U_{e} \approx 0.3 \%$.

After utilizing synthetic images, the same interrogation windows were applied on the experimental data of run 7 . Profiles in wall-normal direction were extracted at the midpoints in streamwise direction of the corresponding $2 \mathrm{D}$ vector fields. All experimental profiles nearly collapsed, independent of interrogation window size, and the differences fell well below the corresponding uncertainties. Therefore, the interrogation window $8 \times 512$ pix $^{2}$ was chosen to perform all following mean velocity analyses due to its high resolution in the wall-normal direction.

Wall temperature Due to the chosen wind tunnel model material PEEK, the wall temperature rose during a wind tunnel run by approximately $3-10 \%$, corresponding to a viscosity change of approximately $2-9 \%$. This influence was analyzed based on numerical calculations with changing wall temperatures, according to the wind tunnel tests. An ensemble averaged velocity field from all temperature computations was compared to the velocity from a single calculation with a mean wall temperature. This resulted in an estimated maximal difference at MS1 of $\Delta U / U \approx 0.5 \%$, limited to a region close to the wall for approximately $y^{+}<20$ $(y / \delta<0.1)$. This difference was included in the uncertainty for the detailed analysis of viscous sublayer velocities (gradient approach for the friction velocity). For larger wallnormal distances, including the logarithmic layer (relevant, e.g, for the fitting for the friction velocity (see Sect. 4.3)), the difference dropped below $\Delta U / U \approx 0.02 \%$ and was therefore neglected.

Particle slip The finite particle response can have a major influence on velocities measured via PIV, especially in low density, hypersonic flows (Ragni et al. 2011; Williams 2014). Simulations were performed with the particle module described in Sect. 2.2.1 to approximate the impact on the velocity profiles in the boundary layer under current experimental conditions. Since only manufacturer data for the used particles and no in situ measurements were available, sensitivity studies were performed to investigate the impact of these parameters on the particle response. On the one hand, the manufacturer values of the applied KRONOS 1002 particles for diameter and density were taken with $d_{p 1}=0.23 \mu \mathrm{m}$ and $\rho_{p 1}=3800 \mathrm{~kg} / \mathrm{m}^{3}$, as representative values for a state without any agglomeration. On the other hand, particle parameters with $d_{p 2}=2.0 \mu \mathrm{m}$ and 
$\rho_{p 2}=800 \mathrm{~kg} / \mathrm{m}^{3}$ were used, according to Williams (2014), approximating an agglomerated state. Williams derived these values experimentally for comparable $\mathrm{TiO}_{2}$ particles at comparable particle Mach and Reynolds numbers. The particles were seeded into the TAU RSM flow solution at $\mathrm{FC} 1$ and $T_{w}=360 \mathrm{~K}$ (see Sect. 4.2) and caught in a plane, corresponding to MS1. Figure 2 contains the modulus of all relative particle velocity components $\left|\mathbf{V}_{\text {rel }}\right|=\left(U_{\text {rel }}^{2}+V_{\text {rel }}^{2}\right)^{0.5}$ (where, e.g., $U_{\text {rel }}=U_{p}-U$ ), based on the streamwise edge velocity component $U_{e}$ to $\left|\mathbf{V}_{\text {rel }}\right| / U_{e}$ for each caught particle $p_{1}$ and $p_{2}$ against the wall-normal distance, normalized by the local boundary layer thickness $\delta$. The collected particles show negligible particle slip outside and near the boundary layer edge, whereas an increase toward the wall is visible. The maximum values are reached with $\left|\mathbf{V}_{\text {rel }}\right| / U_{e} \approx 0.1 \%$ for particle $p_{1}$ and $\left|\mathbf{V}_{\text {rel }}\right| / U_{e} \approx 2.1 \%$ for particle $p_{2}$. The difference in velocity is mainly driven by only weak successive deceleration of high-speed particles through the low-density boundary layer region. Although first decelerated by passing the bow shock in front of the cone, high Knudsen numbers and inertia effects lead to a persisting higher velocity than the surrounding fluid. This difference decreases with increasing running length, visible in Fig. 2 for the second measurement section MS2. Here the maximum relative velocity drops to $\left|\mathbf{V}_{\text {rel }}\right| / U_{e} \approx 1.5 \%$ for particle $p_{2}$.

Although on a noticeable level, the difference due to slip is not included in the general PIV uncertainty but kept in mind for later discussions. This decision was mainly based on the fact that only sensitivity values for the particle

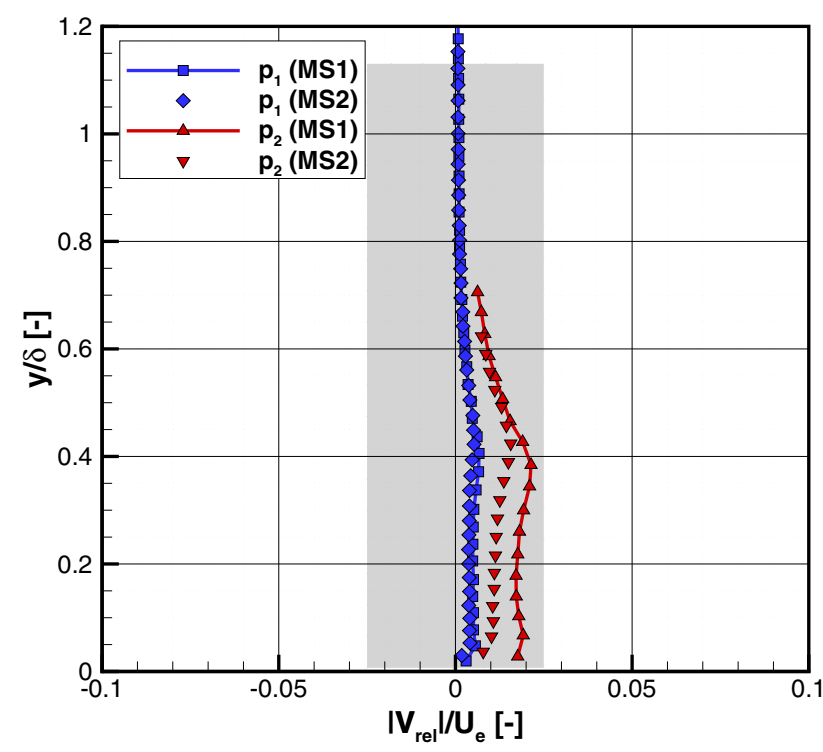

Fig. 2 Relative velocity difference of particles $p_{1}$ and $p_{2}$ along the $7^{\circ}$ cone at FC1 at measurement section MS1 and MS2 (shaded region corresponds to PIV setup velocity uncertainty) parameters were available and so only a tendency could be estimated. Additionally, PIV measurements by Williams et al. (2015) and Williams et al. (2018), using comparable particles in a $M a \approx 7$ boundary layer flow, show no sign of slip influence on the mean velocity profiles, at least for a region including and above the logarithmic layer.

Image number convergence To check the convergence of the mean velocities, the cumulative mean of the streamwise velocity for different amounts of images $N$ was analyzed at three positions outside the boundary layer. The extracted data between $y / \delta=1.59-1.72$, including its uncertainties, showed an agreement with the corresponding Taylor-Maccoll edge velocity as reference for image numbers approximately $N>25$ and nearly constant behavior above $N \cong 150$.

\subsubsection{Comparison to literature data}

Two Pitot probe profiles at measurement section MS1 and inflow condition FC2, processed to Mach numbers according to the procedure described in Sect. 3.2.2, are shown in Fig. 3. The Pitot measurements include a Mach number boundary layer region from approximately $M a=2$ to an edge value of approximately $M a=5.35$, which is in good agreement with the nominal Taylor-Maccoll value for FC2, included as a black line (see also Table 1).

In Fig. 4, the velocities of the two PIV profiles at FC1 (run 7 and 8) are directly compared to the velocities derived from two Pitot pressure runs at FC2 (run 32\&33 and 39\&52), all extracted at the first measurement section MS1. Uncertainties are visualized by a gray area in case of the PIV and by error bars in case of the Pitot data (derived by propagation of uncertainties to $\pm 1.7 \%$ of the edge velocity). Additionally,

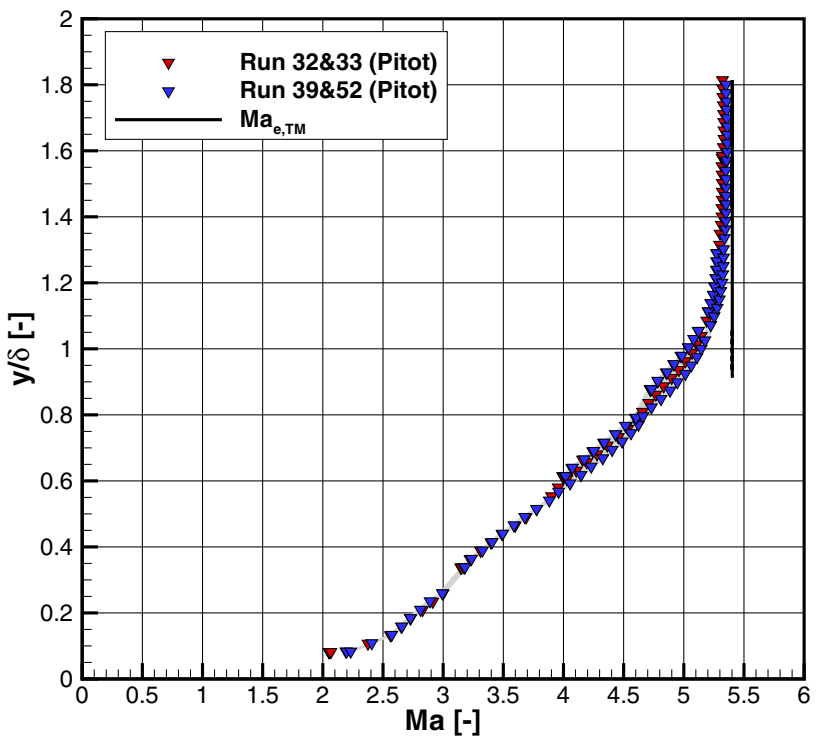

Fig. 3 Mach profiles of Pitot run32\&33 and 39\&52 


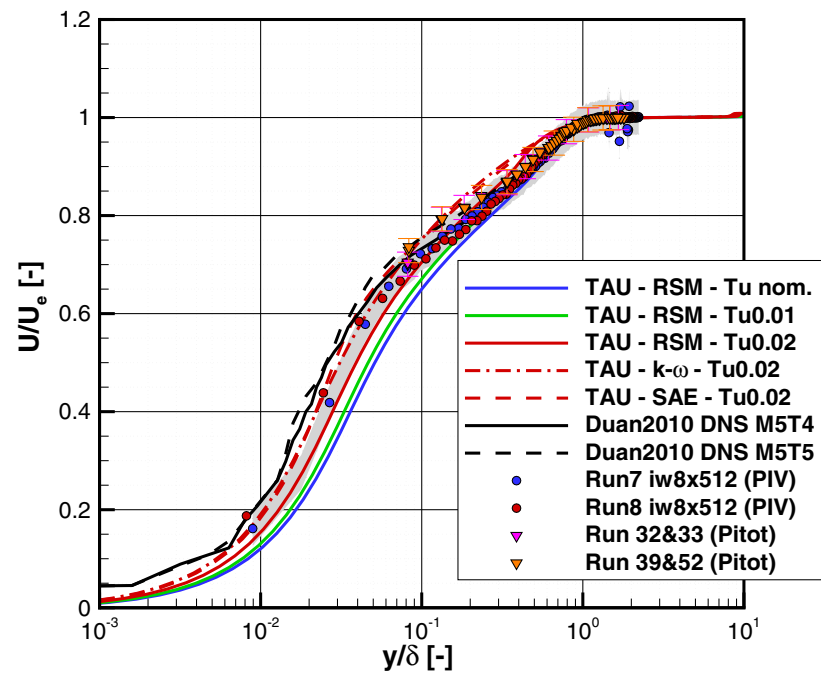

Fig. 4 Streamwise mean velocity profiles from PIV (iw8 $\times 512$ pix $^{2}$ ) and from Pitot pressure at MS1 compared to numerical results (only every second experimental data point plotted for clarity, shaded region corresponds to PIV setup uncertainty, DNS data according to Duan et al. 2010)

different numerical data are included for comparison. TAU computations with different turbulence models (SAE, k- $\omega$ and RSM) and three different inflow turbulent intensity levels are included at $0.1 \%$ (Tu nom., since it corresponds to the nominal value in TAU), $1 \%$ (Tu0.01) and 2\% (Tu0.02). The latter two values represent a sensible range for the $\mathrm{H} 2 \mathrm{~K}$ facility, based on experiences in previous test campaigns and a turbulence characterization campaign with Laser 2 Focus (L2F). Additionally, DNS data of two turbulent boundary layer profiles along a flat plate at two different wall temperatures are included according to Duan et al. (2010). Both DNS profiles share the edge Mach number of $M a_{e}=4.97$, but the Reynolds numbers are $\operatorname{Re}_{\theta}=3819\left(\operatorname{Re}_{\delta 2}=1526\right)$ and $R e_{\theta}=4840\left(R e_{\delta 2}=1537\right)$ for case M5T4 and M5T5, respectively. The corresponding wall to adiabatic wall temperature ratios are $T_{w} / T_{a w} \approx 0.7$ and $T_{w} / T_{a w}=1.0$. In comparison, the Reynolds numbers extracted from the current experimental data are with $R e_{\delta 2}=635-1053$ slightly lower. The corresponding wall temperature condition is with $T_{w} / T_{a w} \approx 0.8$ enclosed by the M5T4 and M5T5 data set. The skin friction coefficient of case M5T4 agrees with $c_{f}=1.5 \times 10^{-3}$ close to the corresponding value of run 7 (see Table 4). All data are plotted in outer scaling.

The Pitot data as well as PIV data show excellent repeatability behavior within their corresponding uncertainty bands. In the upper parts of the boundary layer, above $y / \delta>0.3$, both measurement techniques show good agreement. For smaller wall distances, the Pitot data result in slightly higher values compared to the PIV data, although still covered by the corresponding uncertainties. A good agreement is visible for the upper part between experimental data and TAU computations, if the uncertainties are considered. Results with increased turbulence level of 1\% (Tu0.01) and RSM turbulence model show best agreement, although deviations above the uncertainty band are visible below $y / \delta \approx 0.1$. Sources for this difference can be manifold. Possible contribution can arise from particle slip and increased shear close to the wall, previously identified as influencing factor for the PIV data. Despite the difference in Reynolds number, the DNS and experimental velocity profiles agree within the corresponding measurement uncertainties, except for a region between $0.05<y / \delta<0.01$. This difference might be attributed to the difference in Reynolds number. The data of the second measurement section MS2 shows the same agreement between PIV and Pitot data (not shown here).

\subsubsection{Friction velocity and inner scaling}

To follow the suggested analyses given in the AGARD report 253 (Fernholz and Finley 1980), both Pitot pressure and PIV velocity profiles were transformed to inner and outer scaled form. Three approximations were applied and compared to extract the necessary skin friction velocity indirectly from the measured mean velocity profiles (see Sect. 3.4).

For this skin friction evaluation, different boundary layer edge and wall values were necessary for all approaches. These parameters were extracted from either the velocity data or infrared data and are summarized separately in Table 4 for all investigated wind tunnel runs. The table

Table 4 Edge and wall parameters of fitted Pitot and PIV $\left(8 \times 512\right.$ pix $^{2}$ interrogation window $)$ profiles at FC1 and FC2

\begin{tabular}{|c|c|c|c|c|c|c|c|c|c|c|c|c|c|}
\hline Run No. (-) & MS (-) & $M a_{e}(-)$ & $U_{e}(\mathrm{~m} / \mathrm{s})$ & $T_{e}(\mathrm{~K})$ & $T_{w} / T_{a w}(-)$ & $\delta(\mathrm{mm})$ & $\theta(\mathrm{mm})$ & $H_{12 k}(-)$ & $\operatorname{Re}_{\theta}(-)$ & $R e_{\delta 2}(-)$ & $c_{f}(-)$ & $U_{\tau}(\mathrm{m} / \mathrm{s})$ & $\Pi(-)$ \\
\hline 7 & 1 & 5.03 & 916 & 83 & 0.79 & 3.4 & 0.155 & 1.5 & 2355 & 635 & $1.57 E-03$ & 53.8 & 0.27 \\
\hline 8 & 1 & 5.59 & 927 & 69 & 0.80 & 3.7 & 0.151 & 1.5 & 3298 & 736 & $1.26 E-03$ & 53.7 & 0.41 \\
\hline 10 & 2 & 4.86 & 912 & 88 & 0.80 & 4.2 & 0.204 & 1.6 & 2602 & 743 & $1.48 E-03$ & 50.5 & 0.43 \\
\hline $32 \& 33$ & 1 & 5.33 & 901 & 78 & 0.79 & 3.9 & 0.154 & 2.7 & 3198 & 777 & $1.44 E-03$ & 52.9 & 0.18 \\
\hline $39 \& 52$ & 1 & 5.36 & 902 & 78 & 0.79 & 3.9 & 0.152 & 2.7 & 3191 & 768 & $1.43 E-03$ & 52.9 & 0.20 \\
\hline $13 \& 23$ & 2 & 5.23 & 898 & 82 & 0.79 & 4.9 & 0.207 & 2.3 & 4323 & 1084 & $1.29 E-03$ & 49.1 & 0.33 \\
\hline $24 \& 25$ & 2 & 5.23 & 898 & 82 & 0.79 & 4.8 & 0.202 & 2.5 & 4194 & 1053 & $1.32 E-03$ & 49.6 & 0.29 \\
\hline
\end{tabular}


includes the edge velocity $U_{e}$, the corresponding temperature $T_{e}$ from the Crocco-profile and additionally the corresponding Reynolds numbers $\operatorname{Re}_{\theta}$ and $R e_{\delta 2}$ at the profile positions. The derived edge properties are consistent with each other within the corresponding uncertainties. The resulting Reynolds numbers are $R e_{\theta}=2355-3298$ and $R e_{\delta 2}=635-743$. According to the difference between the inflow condition FC1 and FC2, slightly different edge parameters were derived. This is in agreement with the expected values from Taylor-Maccoll for FC2 (see Table 1). The results of the three approaches to derive the skin friction velocity, the fitting, gradient and integral approach, are summarized and compared in Table 5. Where possible, the values include the corresponding uncertainties, stated in the following paragraphs. In case of the PIV data, only results from the interrogation window $8 \times 512$ pix $^{2}$ are contained.

In general, very consistent skin friction velocities were extracted with an agreement between the different approaches and analytical and numerical data for each measurement section (MS) and flow condition (FC) if the corresponding uncertainties are accounted for (Table 5). The van Driest correlation was used for the analytical (see Sect. 2.1) and the TAU RSM results at $1 \%$ inflow turbulence intensity (see Sect. 2.2) for the numerical comparison.
Fitting approach The skin friction velocities for run 7 and 8 show a good repeatability and the expected decrease for an increased running length for run 10 (Table 5). A slight difference in wall temperature between FC1 and FC2 is the reason for a slight difference in the derived skin friction velocities from Pitot data compared to the corresponding PIV results. This is in agreement with analytical or numerical prediction. The uncertainties for the fitting and integral approach were determined by a Monte Carlo analysis due to the non-linearity of the methods. A sensitivity study showed $N=8000$ samples to be sufficient for a converged result. If not otherwise noted, uncertainties for input parameters were distributed uniformly which represents a conservative approach. The resulting uncertainties are given for a $95.4 \%$ confidence interval. An uncertainty of \pm 0.4 and $\pm 3.4 \%$ of the mean friction velocity was calculated for the Pitot and PIV data, respectively. The above stated values do include only procedural and no methodical uncertainties for the friction velocity. Methodical uncertainties can only be determined by comparing the results to a reference value of higher accuracy, either from experimental, numerical or analytical prediction. Most often, only cross-checks between different methods or comparison to analytical predictions are given in the literature. For the comparable Clauser chart method, which utilizes the velocity data and its slope only in the

Table 5 Summary of derived skin friction velocities at FC1 and FC2 and MS1 and MS2 [all values in (m/s)]

\begin{tabular}{|c|c|c|c|c|c|c|c|}
\hline MS & $\mathrm{FC}$ & Run no. & Fitting approach & Gradient approach & Integral approach & TAU & van Driest \\
\hline \multirow[t]{5}{*}{1} & 1 & & & & & $51.6 \pm 2.9$ & 52.7 \\
\hline & & 7 & $53.8 \pm 1.8$ & $54.4 \pm 4.9$ & & & \\
\hline & & 8 & $53.7 \pm 1.8$ & $54.2 \pm 4.9$ & & & \\
\hline & & $7-10$ & & & $50.7 \pm 17.0$ & & \\
\hline & & $8-10$ & & & $54.5 \pm 18.3$ & & \\
\hline \multirow[t]{7}{*}{1} & 2 & & & & & $50.1 \pm 2.9$ & 51.2 \\
\hline & & $32 \& 33$ & $52.9 \pm 0.2$ & & & & \\
\hline & & $39 \& 52$ & $52.9 \pm 0.2$ & & & & \\
\hline & & $32 \& 33-13 \& 23$ & & & $52.9 \pm 3.0$ & & \\
\hline & & $32 \& 33-24 \& 25$ & & & $51.0 \pm 2.9$ & & \\
\hline & & $39 \& 52-13 \& 23$ & & & $53.5 \pm 3.1$ & & \\
\hline & & $39 \& 52-24 \& 25$ & & & $51.6 \pm 3.0$ & & \\
\hline \multirow[t]{4}{*}{2} & 1 & & & & & $50.4 \pm 2.9$ & 51.4 \\
\hline & & 10 & $50.5 \pm 1.7$ & $51.8 \pm 4.7$ & & & \\
\hline & & $7-10$ & & & $49.2 \pm 16.5$ & & \\
\hline & & $8-10$ & & & $52.6 \pm 17.7$ & & \\
\hline \multirow[t]{7}{*}{2} & 2 & & & & & $49.0 \pm 2.8$ & 50.0 \\
\hline & & $13 \& 23$ & $49.1 \pm 0.2$ & & & & \\
\hline & & $24 \& 25$ & $49.6 \pm 0.2$ & & & & \\
\hline & & $32 \& 33-13 \& 23$ & & & $51.1 \pm 2.9$ & & \\
\hline & & $32 \& 33-24 \& 25$ & & & $49.5 \pm 2.8$ & & \\
\hline & & $39 \& 52-13 \& 23$ & & & $51.7 \pm 3.0$ & & \\
\hline & & $39 \& 52-24 \& 25$ & & & $50.0 \pm 2.9$ & & \\
\hline
\end{tabular}


logarithmic layer, typical uncertainties of approximately $5 \%$ are given in the literature for incompressible investigations (e.g. Purtell et al. 1981; So et al. 1994; Wei et al. 2005). For compressible investigations, the performance is typically stated within approximately $10 \%$ compared to analytical predictions like van Driest (e.g., Berg 1977; So et al. 1994; Williams 2014; Peltier et al. 2016; Williams and Smits 2017; Williams et al. 2018). This is in agreement with observations in this paper (see Table 5).

Gradient approach The gradient approach was possible to apply due to the wall-normal resolution of the $8 \times 512$ pix $^{2}$ PIV profiles with the first point above the wall at $y^{+}(1)=1.79$ which is located within the viscous sublayer (see Fig. 5b). Typically, all skin friction velocity values derived by the gradient approach below $y^{+}=15$ agreed with the corresponding values from the fitting approach. This trend was detected for data from all interrogation windows reported above. Only the wall nearest point in case of the $8 \times 512$ pix $^{2}$ profile was biased. Therefore, reported skin friction values in Table 5 were derived by averaging all values within $8<y^{+}<15$. The uncertainties for the gradient approach were evaluated based on linear propagation to $\pm 9.1 \%$ based on a mean value.

Integral approach For the numerical integration of the von Karman equation, a step size of $0.1 \%$ of the difference between $\theta$ at MS1 and MS2 was set by default. A systematic change of this step size showed a change of the resulting skin friction coefficient of only $\Delta c_{f, e}<0.05 \%$. In case of the PIV data, two different combinations and in case of the Pitot data four different combinations were possible due to repeatability runs. The results, contained in the column named "integral" in Table 5 show slight variations between the possible combinations at each flow condition. The corresponding values still agree with each other and values from the other approaches if the corresponding uncertainties are considered. The combined uncertainties for the integral approach were evaluated to \pm 33.5 and $\pm 5.7 \%$ for the PIV and Pitot data, respectively. The higher uncertainty values in case of the integral approach from PIV data can be attributed to higher input uncertainties of edge Mach number, temperature and momentum thickness.

Due to the low uncertainty and consistent results, it was decided to use the skin friction and Coles wake parameter from the fitting approach to scale and analyze the PIV and Pitot profiles according to Fernholz and Finley (1980). The result for this scaling of the PIV data with $8 \times 512$ pix $^{2}$ of run 7 at MS1 is included in Fig. 5. The figure contains four different sub-plots a to d. In a, the profile is shown as normalized velocity $U / U_{e}$ against normalized wall-normal distance $y / \delta$ for a zoomed region close to the wall. Additionally, a linear line is included which is fitted through data points $y / \delta<0.04$. In sub-plot $\mathrm{b}$, the classical law-of-thewall with the van Driest transformed effective velocities $U_{\text {eff }}^{+}$ against $y^{+}$is included with one line for the viscous sub-layer
Fig. 5 Streamwise mean velocity data from PIV (iw8 $\times 512$ pix $^{2}$ ) and Pitot pressure in inner and outer scaling according to Fernholz and Finley (1980) (DNS data according to Duan et al. (2010))
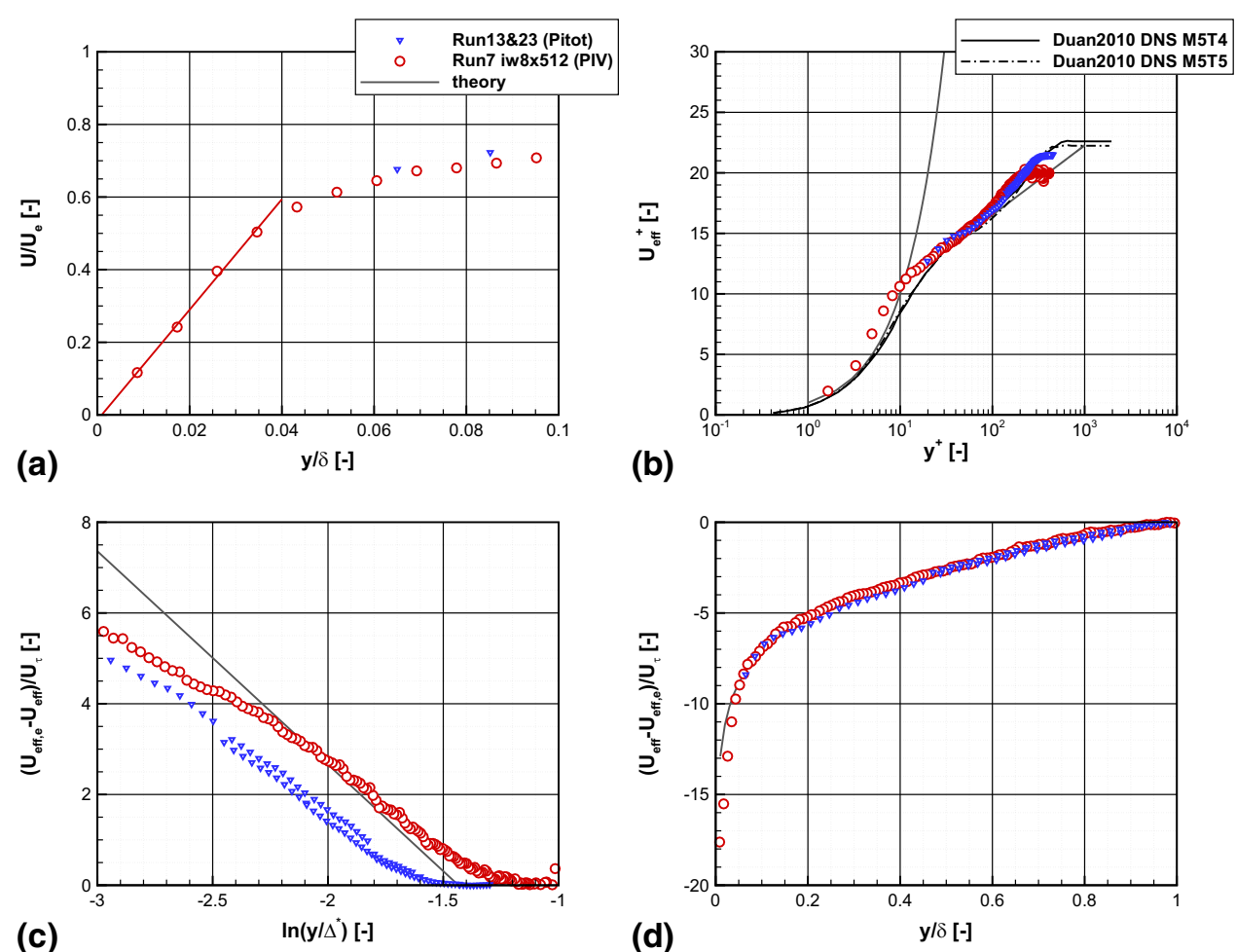
$\left(U_{\text {eff }}^{+}=y^{+}\right)$and one line for the log-layer without wake (see Eq. 1, neglecting the last term). Additionally, the two DNS profiles M5T4 and M5T5 according to Duan et al. (2010) are included in van Driest transformed inner-scaled form. In sub-plot c, an outer scaling is included with the dimensionless velocity defect as $\left(U_{\text {eff,e }}-U_{\text {eff }}\right) / U_{\tau}$ against a wallnormal distance $y$ normalized by an integral length scale $\Delta^{*}$ according to Fernholz and Finley (1980) and defined by

$\Delta^{*}=\delta \int_{0}^{1}\left(\frac{\left(U_{\mathrm{eff}, e}-U_{\mathrm{eff}}\right)}{U_{\tau}}\right) d\left(\frac{y}{\delta}\right)$

The theoretical curve in this sub-plot corresponds to the following equation:

$\frac{\left(U_{\mathrm{eff}, e}-U_{\mathrm{eff}}\right)}{U_{\tau}}=-M \cdot \ln \left(\frac{y}{\Delta^{*}}\right)-N$,

where $M=4.7$ and $N=6.74$, which were derived based on experimental data in zero-pressure gradient boundary layers, mainly along adiabatic walls (Fernholz and Finley 1980). The constants are strictly just valid for $1500<R e_{\delta 2}<40000$, but Fernholz and Finley (1980) showed also consistent analysis for data with Reynolds numbers below this range. In the fourth sub-plot, labeled d, a second outer scaling, represented by $\left(U_{\text {eff }}-U_{\text {eff,e } e}\right) / U_{\tau}$ against $y / \delta$, is included. The theoretical curve is described, as given in Ekoto et al. (2008), by

$\frac{\left(U_{\mathrm{eff}}-U_{\mathrm{eff}, e}\right)}{U_{\tau}}=\frac{1}{\kappa} \cdot \ln \left(\frac{y}{\delta}\right)-\frac{2 \Pi}{\kappa}\left[1-\sin ^{2}\left(\frac{\pi}{2} \frac{y}{\delta}\right)\right]$.

The quality of the fitting procedure is directly visible by comparing the data points to the theoretical curves of the law-of-the-wall in Fig. 5b. All characteristic boundary layer parts are visible, like the $S$-shaped wake above $y^{+} \approx 100$, the logarithmic layer for $100_{\sim}^{>} y^{+>} 10$ and even the viscous sublayer for data points with $y^{+}<10$. The first data point is located well within the viscous sublayer. The linear characteristic of the sublayer is also visible in sub-plot a, comparing the line fit to the wall nearest data points. If compared to the DNS data in sub-plot b, a typical buffer layer is not visible for the current PIV data. One reason might be the particle slip, which was previously identified to be responsible for locally higher velocities in the near wall region (see paragraph particle slip in this section and Fig. 2). The wake portion of the run 7 profile was fitted best by a Coles wake parameter of $\Pi=0.27$ (see Table 4). Due to the low Reynolds number, the wake parameter is lower than $\Pi=0.55$, which is typically stated for an incompressible zero pressure gradient flow. This is well in line with data of other compressible flow investigations in a comparable Reynolds number regime (Fernholz and Finley 1980).
The outer scaling results of the run 7 data are contained in Fig. 5c, d. The theoretical curve in sub-plot $d$ also depends on the wake parameter, so that an agreement with experimental data is a further consistency check. An excellent agreement between theory and data is visible down to wall-normal distances of $y / \delta \approx 0.05$, corresponding to the region of the viscous sublayer (see sub-figure a) for which the theory is not applicable any more. The outer scaling in sub-figure $\mathrm{c}$ shows good agreement between theory and data down to values of $\ln \left(y / \Delta^{*}\right) \approx-2$. For lower wall distances, the data points deviate from theory which was reported for low Reynolds number profiles at $R e_{\delta 2}<2000$ in Fernholz and Finley (1980). This is consistent with the value range in this paper. To check if the flow is fully turbulent, despite low Reynolds numbers, the kinematic shape factor is a useful indicator, defined as the ratio of displacement and momentum loss thickness with the density set constant to $H_{12 k}=\delta_{k}^{*} / \theta_{k}$. The PIV profile of run 7 with $H_{12 k} \approx 1.5$ (see Table 4) lies in a value range characteristic for fully turbulent boundary layers at low to moderate Reynolds numbers (Fernholz and Finley 1980).

The same analysis was performed for velocity data derived from Pitot pressures of run $13 \& 23$, also included in Fig. 5. Although small, the dimension of the Pitot probe restricts the profile to a first wall-normal distance of $y(1)=0.32 \mathrm{~mm}, y / \delta(1)=0.082, y^{+}(1)=19.5$ and therefore almost one order of magnitude farther away from the wall, compared to PIV profiles. This is directly visible in subplot $b$, where the profile begins in the logarithmic part of the boundary layer. A small region of locally higher velocity values compared to PIV and theory is visible. This was already visible in Fig. 4 and is most likely connected to near wall effects of the Pitot probe (Grosser (1996)), also visible in other investigations like Ekoto et al. (2007) or Sahoo et al. (2009). Besides this region, the profile shows acceptable agreement with the theory in the logarithmic layer. The outer scaled data in sub-plot $\mathrm{d}$, which is driven by parameters like $y / \delta$ and $\Pi$, shows very good agreement with theory. Instead, the outer scaling in sub-plot c, driven by an integral length scale and therefore by near wall data, is parallel shifted from the theoretical line. This is mainly driven by the loss of near wall information due to the Pitot probe dimension. This is also noticeable with a shape factor of $H_{12 k}=2.3$ (see Table 4), which is close to the value of a Blasius profile with $H=2.59$ and would erroneously indicate a laminar profile (White 2006). To support this argument, a check was performed with an an artificial profile, derived by the law-ofthe-wall, which was scaled to an edge velocity according to $\mathrm{H} 2 \mathrm{~K}$ conditions. Before using this artificial profile for the fitting analysis, the profile was intentionally cut below $y / \delta=0.08$, comparable to the Pitot data. The corresponding fitting results showed the same shift for the integral based profiles, as visible in Fig. 5c. 
With the reported measurement techniques, a total number of approximately 10 and 10-30 points in the logarithmic layer are available for analysis from Pitot and PIV data, respectively. Additionally, the general agreement between the different skin friction velocity approaches and theory and numerics give confidence for a future analysis of rough wall data.

\subsection{Turbulence data}

Turbulence intensities were analyzed based on the PIV data. First, the impact of several influences and uncertainties is discussed. After that, data for the streamwise and wallnormal turbulence intensities are compared to literature and numerical data and the applicability of Morkovin's hypothesis is tested.

\subsubsection{Uncertainties and sensitivities}

Several different influences can affect the extraction of turbulence intensities via PIV measurements (see, e.g., Adrian and Westerweel 2011). The influence of random errors, spatial resolution and particle lag filtering and convergence with image number has been analyzed and is discussed in the following.

Random error The impact of random errors was analyzed for selected interrogation windows. A strategy described in Williams (2014) and originally based on a work by Discetti et al. (2013) was followed, which utilizes two-point correlations in the streamwise direction derived from the instantaneous velocity vectors of all recorded images. It is based on the assumption that random PIV measurement noise is statistically uncorrelated between non-overlapping interrogation windows. Therefore, information from the correlation curve with a distance $\Delta x$ greater than the overlap can be used to extrapolate an unbiased value at zero distance. In this work, a linear fit was used, which tends to underestimate the error (Williams 2014), but an alternative parabolic fit was not feasible due to resolution.

Unfortunately, no random error could be extracted directly for the high aspect ratio interrogation windows with a streamwise extent of 512 pixel (as was used for the previous mean velocity discussion), since no non-overlapping points are available for the two-point correlation within the current ROI. Instead, a multigrid approach starting from $512 \times 512 \mathrm{pix}^{2}$ to a final size of $i y \times 256 \mathrm{pix}^{2}$, with $i y$ for different wall-normal dimensions, was used to approximate the error. Due to non-consistent two-point correlation curves, the results for windows with $8 \times 256$ pix $^{2}$ and therefore also $8 \times 512$ pix $^{2}$ were discarded for further analyses.

At the boundary layer edge, windows with $16 \times 256 \mathrm{pix}^{2}$, $32 \times 256$ pix $^{2}$ and $64 \times 256$ pix $^{2}$ result in random errors of approximately 27,28 and $12 \%$ of the local turbulence intensity, respectively. For the same interrogation windows, the analysis for points in the boundary layer resulted in random errors of approximately $27,19,13 \%$ at $y / \delta \approx 0.8$ and 65 , $67,27 \%$ at $y / \delta \approx 0.5$. For a reference value, a large interrogation window with a final size of $256 \times 256$ pix $^{2}$ was used, restricted to the boundary layer edge due to its size. This window resulted in a random error of $5 \%$. This decrease of error with increasing window size could imply that the amount and distribution of particles in the flow is only sufficient for a moderate quality of turbulence data. Before a decision was made on the selection of window size, the subgrid filtering was analyzed.

Sub-grid filtering The drawback of using larger interrogation windows to reduce random errors is an increased amount of sub-grid filtering. In this paper, this effect was estimated based on the work by Spencer and Hollis (2005), who performed analyses with synthetic and experimental data. Spencer and Hollis compared their results to theoretical curves by Host-Madsen and McCluskey (1994), who based their work on the assumption of homogeneous isotropic turbulence for particles within a single interrogation window. Both works investigated the impact of interrogation window size, but exclusively for an aspect ratio $A R=1$. Since in this paper windows with an aspect ratio $A R \neq 1$ were used, this influence on the sub-grid filtering was analyzed separately, following the synthetic data approach by Spencer and Hollis (2005). Synthetic velocity fields with pre-defined mean velocities, Reynolds stresses and two-point correlations were created by applying digital filtering based on a technique introduced by Klein et al. (2003). The sub-grid filtering is approximated by spatial averaging the synthetic velocity field, where the corresponding spatial box represents a simplified approximation of an interrogation window. This approach just simulates the filter characteristics without being influenced by typical additional factors of PIV analysis like, e.g., particle density or size distribution. See Spencer and Hollis (2005) and Klein et al. (2003) for further details of this approach. At this point, the symmetrical spatial box filter for averaging originally implemented by Spencer and Hollis (2005), was extended to an asymmetrical box, approximating interrogation windows with different aspect ratios. The approach was validated for windows with $A R=1$ with the data of Spencer and Hollis (2005). Typically, inputs like mean velocities and Reynolds stresses were used as reported in Spencer and Hollis (2005). Additionally, values for mean velocities, Reynolds stresses, field size and number of fields were varied and the influence on the results was found to be negligible.

The synthetic two-point correlation was prescribed by an exponential distribution, defined by a prescribed integral length scale $L_{u^{\prime} u^{\prime}}$ (see Spencer and Hollis (2005)). A value for this length was based on literature data, since the limited PIV ROI and resolution of the data in this paper prohibited 
an extraction from the data directly. The sub-grid filtering analysis in this paper was first performed at the boundary layer edge. Here, a rough estimate for a reference turbulence intensity value could be used for comparison, based on the L2F measurements, previously mentioned.

First, a lower value with $L_{u^{\prime} u^{\prime}}=0.5 \delta$ was assumed, based on data by Peltier (2013). The author investigated a $M a=4.9$ turbulent boundary layer at higher Reynolds number $R e_{\theta}=23000\left(R e_{\delta 2}=5300\right)$, but comparable wall temperature with $T_{w} / T_{a w}=0.93$ via PIV measurements. The author approximated the area below the two-sided twopoint correlation curve assuming an exponential behavior and extracting the length where this curve falls below a value of $1 / e$. Second, an upper value with $L_{u^{\prime} u^{\prime}}=3 \delta$ was used, based on data by Ringuette et al. (2006). The authors investigated turbulent boundary layers from $M a=3-5$ at Reynolds numbers ranging from $R e_{\theta}=2390-6225$ and adiabatic wall temperatures via DNS computations. The integral length for all Mach numbers are reported throughout the boundary layer, extracted by integrating the corresponding two-point velocity correlation curves. At the boundary layer edge $L_{u^{\prime} u^{\prime}} \approx 1.5 \delta$ and $L_{u^{\prime} u^{\prime}} \approx 1.25 \delta$ is reported for $M a=3$ and $M a=5$, respectively. For the lower Mach number case, the authors investigated the influence of domain size by increasing the streamwise extent by a factor of 2.6, which resulted in a similar increase of the corresponding integral length scale. This indicates a length scale truncation due to limited region for integration, typically occurring for, e.g., PIV measurements. Therefore the latter $M a=3$ value from the increased domain was chosen as upper value, although a decrease of length scale with increasing Mach number is expected (as visible for the nominal domain size in Ringuette et al. (2006) and discussed in Dussauge and Smits 1997)

Based on the lower length scale of $L_{u^{\prime} u^{\prime}}=0.5 \delta$, the filtering analysis resulted for the investigated interrogation window sizes of $16 \times 512$ pix $^{2}, 32 \times 512$ pix $^{2}$ and $64 \times 512$ pix $^{2}$ in a resolvable content of 77, 72 and $71 \%$ of the local turbulence intensity at the boundary layer edge, respectively. If those interrogation windows were characterized based on the original square window data by Spencer and Hollis (2005), the resolvable content due to filtering would erroneously result in 53\% for all the above-stated windows, since the streamwise extent of 512 pixel would be the only decisive parameter, independent of the wall-normal extent. The previously defined large control interrogation window with a final size of $256 \times 256$ pix $^{2}$ resulted in resolvable content of $72 \%$. If instead the upper estimate for the integral length scale with $L_{u^{\prime} u^{\prime}}=3 \delta$ is assumed for the filtering analysis, all investigated interrogation windows (except the $256 \times 256$ pix $^{2}$ window) resulted in $94-95 \%$ resolved content.

Based on the collected data for random error and subgrid filtering for selected interrogation windows, a size of $64 \times 512$ pix $^{2}$ was selected for the following discussion. This window represents a trade-off between random error and sub-grid filtering. At the same time, the wall-normal extent enabled measurements within the boundary layer and the difference of the turbulence intensity at the boundary layer edge was kept below approximately $8 \%$ compared to the large control window with $256 \times 256 \mathrm{pix}^{2}$.

So far, the analysis was restricted to the boundary layer edge. The above stated values are most likely underestimating the sub-grid filtering within the boundary layer, since the prediction was based exclusively on outer layer length scales. If the length scale distribution throughout the boundary layer according to Peltier (2013) is used in the modified Spencer approach, as described above, a difference of only $5 \%$ compared to the edge value would be determined for $y / \delta>0.1$. This is mainly due to only small changes in the length scale data, which is most likely also biased since the corresponding values were derived from PIV investigations, which are also affected by resolution and filtering effects. An order of magnitude analysis for the sub-grid filtering within the boundary layer independent of biased length scales can be derived based on an approach according to Lee et al. (2016). The authors derived a database for the attenuation of Reynolds stress, which uses a filter volume, defined by inner-scaled interrogation window size and the laser sheet thickness, as input. This database is based on DNS data of an incompressible turbulent boundary layer at $\operatorname{Re}_{\tau}=u_{\tau} \delta / v_{w}=1500\left(\operatorname{Re}_{\theta} \approx 5000\right)$ according to Sillero et al. (2013). Unfortunately, the database is limited to a streamwise interrogation window size which corresponds to 238 pix under $\mathrm{H} 2 \mathrm{~K}$ setup conditions. Therefore, a window of $64 \times 238$ pix $^{2}$ was used to estimate the streamwise Reynolds stress attenuation according to the procedure described in Lee et al. (2016). As reference, the $M a=4.97$ turbulent boundary layer DNS data according to Duan et al. (2010) was used. For this approximation, inner scaling of the wall-normal distance and Morkovin's scaling of the Reynolds stress of the Duan data were applied. The derived filtering values were compared to approximations from the previously described approach based on Spencer and Hollis (2005) at the same interrogation window, using the integral length scale distribution according to Peltier (2013). The comparison results in a maximum underestimation of resolvable content by the Spencer approach in the order of approximately $6 \%$ of the local rms velocity. This maximum is located at a wall-normal position close to the Reynolds stress peak in the buffer layer.

Additionally, the method according to Lee enables an approximation of the impact of laser sheet thickness on the sub-grid filtering. A maximum further decrease of resolvable rms velocity content of approximately $7 \%$ and approximately $19 \%$ was estimated for a laser-sheet thickness of $0.5 \mathrm{~mm}$ and $1.0 \mathrm{~mm}$ in the $\mathrm{H} 2 \mathrm{~K}$ setup, respectively. These values were derived based on the Duan DNS data and an interrogation 
window of $64 \times 238$ pix $^{2}$ and therefore represent most likely a lower limit for the larger $64 \times 512$ pix $^{2}$ window, used in the following analyses.

Please note that the filtering approximation according to Lee et al. (2016) is based on inner (e.g., interrogation window sizes) and the one according to Spencer and Hollis (2005) was used with outer scaled relevant length scales (e.g., integral length scales). Therefore, a direct comparison between results of both methods is challenging, if the Reynolds numbers $R e_{\tau}$ are not comparable for data sets, from which relevant length scales are used.

Particle slip Independent of the post-processing, the measurement of turbulence intensities is influenced by particle slip for tracer based techniques. The particle slip can have a bigger impact on the turbulent part than on the mean velocities (see, e.g., Williams 2014). To approximate the impact of the particle response in $\mathrm{H} 2 \mathrm{~K}$ conditions, representative Stokes numbers $S_{k}$ were estimated. Defined by $S_{k}=\tau_{p} / \tau_{f}$, the characteristic flow time constant $\tau_{f}$ and particle time constant $\tau_{p}$ are necessary.

To estimate the particle time constant the response through an oblique shock is typically investigated in superand hypersonic flows (e.g., Ragni et al. 2011). Williams (2014) showed that results from those tests may overestimate the capability of particles to follow turbulent disturbances. In case of small disturbances of turbulence the particle response is mainly driven by slip and therefore a function of Knudsen number. Instead, Williams (2014) performed simulations, based on the equation of particle motions, for pre-defined wall-normal positions and their corresponding local flow parameters in the boundary layer. The equation of motion of a particle in a fluid can be simplified based on assumptions like high particle to fluid density ratio and small, spherical particle dimensions (Williams 2014). The resulting equation may be integrated numerically, assuming suitable drag models for the corresponding flow regime. In this paper, this approach was adopted and the drag models stated in Sect. 2.2.1 were implemented. The numerical integration was typically performed with a step size $d t$ of $1 \%$ of the corresponding Stokes relaxation time. Representative particle relaxation times $\tau_{p}$ for all applied drag models were extracted where the normalized velocity ratio drops to $1 / \mathrm{e}$ (Williams 2014). The integration tool was validated with literature data.

In this paper, the required input parameters like mean velocity, density, temperature and viscosity were extracted from a TAU RSM boundary layer profile at $T_{w}=360 \mathrm{~K}$ (MS1, see Table 3). To be comparable with Williams (2014), a simulation with a representative turbulence level of $0.05 U_{e}$ for 40 equidistant wall-normal locations was performed for both particles $p_{1}$ and $p_{2}$, defined in Sect. 4.3. Comparison of this turbulence level to actual PIV results shows that the results should give a reasonable estimate.

The fluid time constant $\tau_{f}$ is typically chosen to represent a characteristic length scale, e.g., the large eddies in the turbulent boundary layer. For the current analysis, a value with $\tau_{f}=10 \delta / U_{e}$ was taken according to Williams et al. (2015) or Williams et al. (2018).

The results for particle $p_{1}$ and $p_{2}$ are contained in Fig. 6 as Stokes numbers along the wall-normal distance in the boundary layer. The corresponding particle relaxation times $\tau_{p}$ were derived by the described simulations for the Stokes and Tedeschi/Henderson drag model (see Sect. 2.2.1). For the latter two, a mean value is given and the error bars represent the differences between both. For the Stokes drag model, Fig. 6 contains only one representative curve for particle $p_{1}$, just to show the difference in slope and level, erroneously resulting in $S_{k}<0.1$, if no Mach and Knudsen effects are considered. If compressible and rarefied influences are included, the results for the Tedeschi/Henderson models span a range between $0.4<S_{k}<1.5$, depending on the particle type. These values violate the limit of approximately $S_{k} \leq 0.1$ which is considered to be adequate not to filter large-scale turbulence based on LDV measurements according to Samimy and Lele (1991). With data from the same authors, an estimate for the amount of filtering of the turbulent intensity is possible, based on the Stokes number. The reduction in streamwise rms turbulence intensity is approximately 8 and $15 \%$ of a reference velocity like $U_{e}$, for particle $p_{1}$ and $p_{2}$, respectively. The latter value is similar

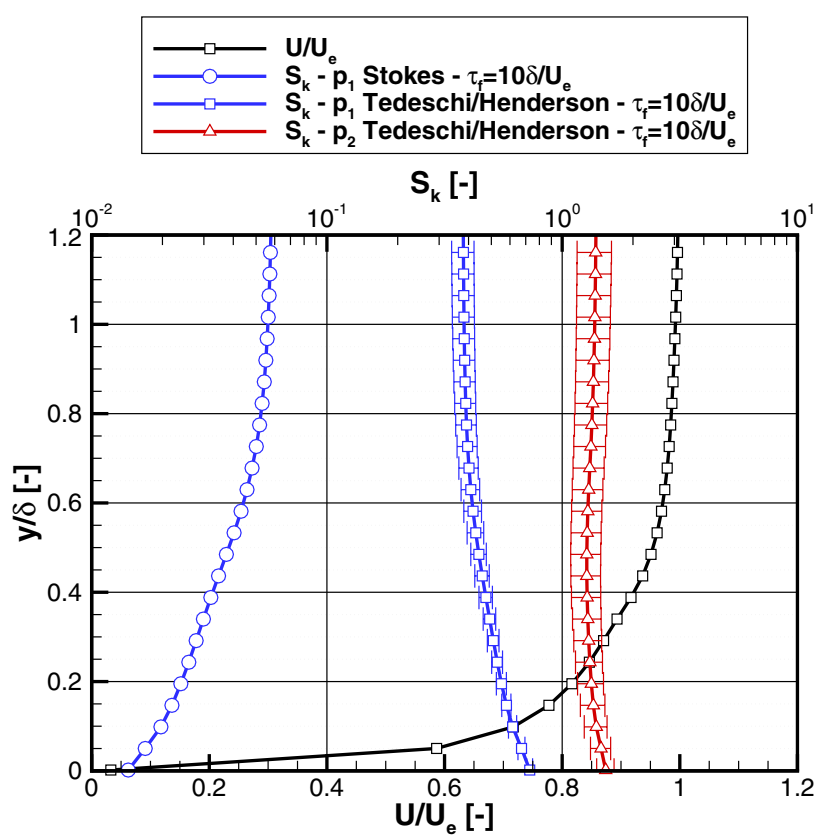

Fig. 6 Variations of Stokes numbers due to turbulence motion across the boundary layer for FC1 
to reductions published for comparable particle and Stokes numbers by Williams et al. (2018). The authors do report about a clear particle lag effect for the wall-normal turbulence intensity but show only limited effect on the streamwise counterpart (see also discussions and comparisons in Sect. 4.4.2).

Image number convergence The uncertainties of the turbulent fluctuations due to convergence were based on the work of Peltier (2013) and used a re-sampling algorithm via the bootstrap method according to Benedict and Gould (1996). Due to only limited number of approximately 200 images per run, data of run 7 and 8 were combined by applying a weighting by the corresponding amount of images per run, according to Sahoo et al. (2009). The estimated convergence results in approximately $8 \%$ of the local streamwise turbulence intensity for the total of 429 images, independent of the used interrogation window.

Additional influences to the above discussed may affect the measurement of turbulence intensities.

\subsubsection{Comparison to literature data}

The turbuelnce intensities are shown in the following in a density-weighted scaling via $\sqrt{\rho / \rho_{w}}$ and friction velocity, as proposed by Morkovin (1962) to account for compressibility. Figure 7 contains the data combined for runs 7 and 8 with the previously characterized interrogation window $64 \times 512$ pix $^{2}$. The uncertainties of the experimental $\mathrm{H} 2 \mathrm{~K}$ data are visualized as gray area and include only the

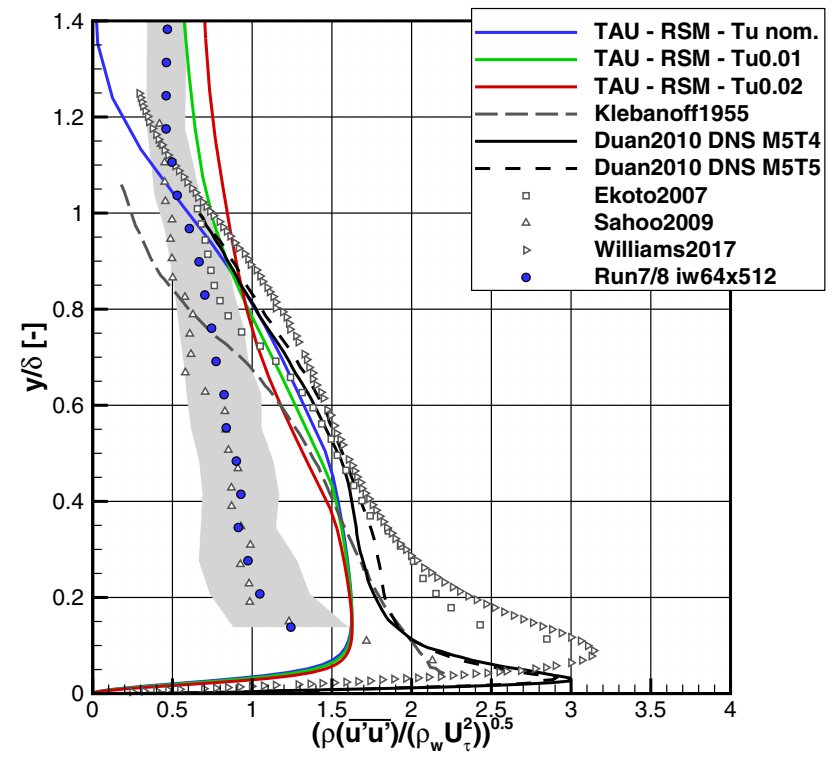

Fig. 7 Density-weighted streamwise turbulence of run 7/8 compared to literature and numerical data after Sahoo et al. (2009), Duan et al. (2010), Williams and Smits (2017), Klebanoff (1955) and Ekoto et al. (2007) (the latter two as reported in Bowersox 2007) uncertainty due to convergence, density and friction velocity from linear propagation. In addition to the TAU RSM data, profiles of DNS calculations according to Duan et al. (2010) are compared (mean velocity profiles were already shown in Fig. 4). For comparison, experimental boundary layer data was included with the $M a=2.86, R e_{\theta} \approx 60000$ data according to Ekoto et al. (2007), $M a=7.2, R_{\theta} \approx 3600$ data according to Sahoo et al. (2009) and $M a=7.6, R_{\theta}=9337$ data according to Williams and Smits (2017). Additionally, incompressible experimental data by Klebanoff (1955) were included $\left(R e_{\theta}=6940\right)$. The profiles of Klebanoff and Ekoto were taken as reported in Bowersox (2007).

The profile of the current data shows the general S-shaped behavior, as visible in the other included data sets. If the uncertainty band is considered, the current data falls slightly below the TAU RSM profile for a turbulence inflow level of $1 \%$ down to the upper part of the boundary layer at $y / \delta \approx 0.6-0.8$. If additionally the approximated amount of random error and sub-grid filtering would be considered, the experimental data points agree within approximately $9 \%$ to the numerical curve. At the boundary layer edge, a difference between all experimental data, including the incompressible data by Klebanoff, is apparent. This behavior might be influenced by a difference in inflow turbulence level of the different wind tunnel facilities (as visible by the shift in TAU profiles) and also by a difference in the definition of the boundary layer edge.

Below $y / \delta \approx 0.6-0.8$, an increased difference between current data and all numerical predictions is visible, which is mainly driven by a turbulence intensity difference but also in part by a slight difference of the corresponding temperature profiles (not shown here). Above $y / \delta \approx 0.2$, TAU RANS and DNS data generally agree, whereas below, a much lower turbulence intensity peak level near the wall is visible for the TAU RANS solutions.

The region of underpredicted turbulence intensities of the current data at $y / \delta \approx 0.6-0.8$ coincides with results from particle response analyses, which showed an increase in slip effects below the corresponding wall-normal distance, resulting in the filtering of accessible fluctuations (see Fig. 6). This underprediction is also visible for the data at $M a=7.2$ (Sahoo et al. 2009) which is likely to suffer from slip influences as well. A lower slip effect is expected for the lower Mach number data at $M a=2.86$ (Ekoto et al. 2007), which agrees much better with numerical turbulence levels in the inner part of the boundary layer. However, more recent data at $M a=7.6$ by Williams and Smits (2017) show much higher turbulence intensities, although measured at Stokes numbers comparable to the $\mathrm{H} 2 \mathrm{~K}$ conditions (if based on a comparable fluid time constant). This contradicts the previous argumentation and would indicate a different or additional reason for the lower values of the current data. 
Another possible reason for underprediction of turbulence stresses is the sub-grid filtering, which was addressed in the sensitivity discussion above (Sect. 4.4.1). It is expected to have an increasing impact with decreasing wall distance, since the dominant length scales of coherent structures typically decrease (e.g., Adrian 2007). Following the approximation after Lee et al. (2016), which included the impact of laser sheet thickness, the most conservative approximation based on a maximum interrogation window $64 \times 238$ pix $^{2}$ and a $1 \mathrm{~mm}$ laser sheet thickness resulted in a maximum resolvable content of $82 \%$ of the local turbulence intensity at a wall distance of $y / \delta \approx 0.2$. The corresponding ratio of measured to DNS intensity at this wall-normal distance is with approximately $60 \%$ clearly below the approximated value. It is expected that the filter analysis with a smaller streamwise window dimension (238 pix) underestimates the filtering of the current windows (512 pix). But comparisons of PIV data between $64 \times 256 \mathrm{pix}^{2}$ and $64 \times 512 \mathrm{pix}^{2}$ windows showed only a difference of approximately $9 \%$ at maximum, which would still not fully cover the difference to the approximated content. Possible influences which might lead to an underprediction in the current filtering analysis are, e.g., the necessary transfer from compressible to incompressible data via Morkovin and inner scaling, the low Reynolds numbers of the DNS and the current PIV data and the assumption of a general similitude of the turbulent structures in axisymmetric and two-dimensional boundary layers, although supported by, e.g., Li et al. (2008). Besides the slip and sub-grid filtering, the seeding quality or other unidentified sources of errors, not covered in the above analyses, might influence the data and analysis as well and contribute to a bias in measured turbulence intensities.

The corresponding wall-normal component of the density-weighted turbulence intensities for the same or comparable data sets are included in Fig. 8. For this plot, the flat plate DNS data is taken from Duan et al. (2011) with slightly different flow conditions than the profiles before with $M a_{e}=4.9, R e_{\theta}=4932, R e_{\delta 2}=1578$. The wall-normal component of a $M a=7.6$ boundary layer at $R e_{\theta}=4940$ is included as published in Williams et al. (2018).

The current data profile (run 7/8) falls below the TAU RSM data at $1 \%$ turbulence inflow in the entire boundary layer. The characteristic peak around $y / \delta \approx 0.2$, visible in all computations, is not visible for the current data. It is the same for the compressible data of the other investigators, where in case of the $M a=7.2$ data by Sahoo et al. (2009), even a decrease is visible. The $M a=7.6$ data at a comparable Reynolds number by Williams et al. (2018) shows a behavior very comparable to the current data. Williams attributed this behavior to the particle slip, since usually measurements of wall-normal turbulence is significantly more sensitive to particle lag than streamwise turbulence in high-speed flows (Williams 2014; Williams et al. 2018).

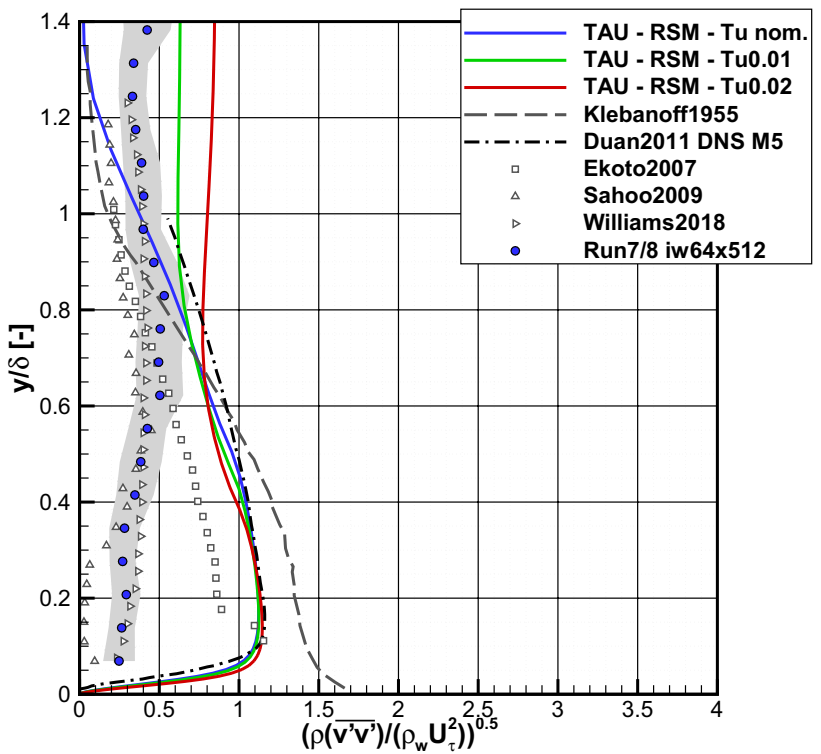

Fig. 8 Density-weighted wall-normal turbulence of run 7/8 compared to literature and numerical data after Sahoo et al. (2009), Duan et al. (2011), Williams et al. (2018), Klebanoff (1955) and Ekoto et al. (2007) (the latter two as reported in Bowersox 2007)

In general, it can be concluded that the prediction of turbulence intensities is very sensitive, even for the same measurement technique and also for numerical predictions. As a result of the overall uncertainties and the deviations between the data, no clear statement could be made about the applicability of Morkovin's scaling based on the current analysis.

\section{Conclusions}

An experimental study was performed to characterize the hypersonic turbulent boundary layer at $M a=6\left(M a_{e}=5.4\right)$ along a $7^{\circ}$ sharp smooth cone model at low Reynolds numbers $R e_{\theta} \approx 3000\left(R e_{\delta 2} \approx 1000\right)$. The applied experimental setup was characterized as a basis for succeeding investigations on rough wall models, with the focus on the mean and friction velocity.

Pitot pressure and particle image velocimetry measurements were used to analyze the velocities in the boundary layer. The Pitot pressure measurements were used to extract velocity profiles with the assumption of a Crocco-Busemann temperature profile. Both measurement techniques were investigated with respect to their sensitivities and uncertainties. In case of PIV, influences like interrogation window size, image number and analysis method were analyzed and discussed. Elongated interrogation windows were suitable to fulfill defined validation criteria. Numerical particle slip simulation estimated a possible considerable amount of relative velocities within the boundary layer, depending on 
the used particles. PIV data was used to extract spatially resolved mean velocity profiles with a resolution down to $\Delta y^{+} \approx 1.8$ at two measurement sections, resolving points within the viscous sub-layer of the thin boundary layer. Both velocity measurement techniques agreed within their uncertainty bands. The experimental mean velocity profiles agreed favorably with numerical RANS data and were also in agreement with DNS profiles of a flat plate flow at comparable Mach number and slightly higher Reynolds numbers (Duan et al. 2010).

Both, Pitot and PIV data, were used to extract the skin friction velocity indirectly. Three different approaches were applied and compared to analytical and numerical data. First, a fitting approach was used based on the incompressible lawof-the-wall. Furthermore, the high resolution of PIV velocity data enabled an approach which approximated the velocity gradient close to the wall. Finally, an integral approach was used which utilized profiles of both measurement sections and the corresponding loss of momentum. All approaches agreed for both experimental techniques with the analytical and numerical predictions within their corresponding uncertainties. Based on Monte Carlo analysis, the fitting approach resulted in the lowest uncertainties and was therefore chosen to enable analysis of the velocity profiles in inner and outer scaling according to AGARD suggestions (Fernholz and Finley 1980). With the van Driest transformation, the compressible velocity profiles generally collapsed with the incompressible law-of-the-wall. Despite that, higher velocities were determined in the buffer layer region, possibly driven by artificially higher velocities due to slip. In outer scaling, the data collapsed in the range of validity with the corresponding theories. Both, outer scaling and shape factor, were biased in case of the Pitot profiles due to the lack of resolution close to the wall. Nevertheless, outer scaling parameters, as well as the skin friction velocities, could be extracted from the Pitot data.

The PIV data allowed analysis of turbulence intensities in the hypersonic boundary layer. Influences like random error, sub-grid-filtering, interrogation window size and convergence with image number were approximated and discussed. Despite the consistent results for the mean velocities, the analysis of turbulence intensities showed higher sensitivities to interrogation window dimensions. Due to the setup conditions, like particle density in the flow, approximated random errors in the order of $30 \%$ and a resolvable content in the order of $70 \%$ due to sub-grid filtering needed to be accepted. Based on these data, a window was chosen and the resulting turbulence intensities were compared to literature data.

If the streamwise turbulence intensities were scaled by the mean density and friction velocity, as suggested by Morkovin, the measured levels are close to TAU RSM calculations in the upper parts of the boundary layer, if typical turbulence inflow intensities of the $\mathrm{H} 2 \mathrm{~K}$ wind tunnel of $T u=1 \%$ were prescribed. In the inner boundary layer, below $y / \delta \approx 0.6-0.8$, the measured turbulence level fell below all numerical predictions, including DNS data at comparable flow conditions. This behavior is in agreement with $M a=7.2$ PIV data by Sahoo et al. (2009), but not with $M a=2.86$ and $M a=7.6$ PIV data by Ekoto et al. (2007) and Williams and Smits (2017), respectively. Although a considerable amount of slip was approximated for the $\mathrm{H} 2 \mathrm{~K}$ conditions, this influence is not likely the main reason for this difference for the streamwise component, since the measurements by Williams and Smits (2017) were performed in a comparable Stokes number regime (if similar fluid time scales were used). Additional uncertainties like influence of laser sheet thickness and sensitivity to the approximation method on sub-grid filtering were addressed, but could not fully explain the missing content of the measured turbulence intensities. The observed reduced content of measured streamwise turbulence intensities in the current data was also visible for the corresponding wall-normal component, with even bigger deviations from numerical predictions. In contrast to the streamwise component, this deviation is visible for all high-speed data included in the current analysis, independent of the author. For the attenuation of the wall-normal component, the slip is typically stated as main contributor in the literature. As a result of the overall uncertainties and the deviations between the data, no clear statement could be made about the applicability of Morkovin's scaling based on the current analysis.

Despite the sensitivities of turbulence intensities, the agreement for the mean and friction velocities between PIV, Pitot and numerical predictions are promising, so that the setup will be used in the next step to analyze the effect of wall roughness on the turbulent boundary layer and also its impact on the heat flux. Improvements are planned for the setup to increase the quality for the extraction of turbulence intensities.

Acknowledgements The authors express their special acknowledgment to Michael Kosbow, Marco Schmors, Viktor Kühl, Vicente Valero Fort and Viola Dehe for their very efficient work with respect to the test preparation, facility operation and support for the PIV and Pitot measurements.

Open Access This article is distributed under the terms of the Creative Commons Attribution 4.0 International License (http://creativeco mmons.org/licenses/by/4.0/), which permits unrestricted use, distribution, and reproduction in any medium, provided you give appropriate credit to the original author(s) and the source, provide a link to the Creative Commons license, and indicate if changes were made. 


\section{References}

Abgrall R, Desideri JA, Mallet M, Periaux J, Perrier P, Stoufflet B (1992) Hypersonic flows for reentry problems. In: Proceedings of the INRIA-GAMNI/SMAI Workshop on Hypersonic Flows for Reentry Problems, Part II, Antibes, France, 15-19 April 1991, Vol III. ISBN 10: 3540561897, 13: 9783540561897

Adrian RJ (2007) Hairpin vortex organization in wall turbulence. Phys Fluids 19(4):041301

Adrian RJ, Westerweel J (2011) Particle image velocimetry, vol 30. Cambridge University Press, Cambridge

Anderson JD (1990) Modern compressible flow: with historical perspective, vol 12. McGraw-Hill, New York

Benedict L, Gould R (1996) Towards better uncertainty estimates for turbulence statistics. Exp Fluids 22(2):129-136

Berg DE (1977) Surface roughness effects on the hypersonic turbulent boundary layer. $\mathrm{PhD}$ thesis, California Institute of Technology

Bowersox R (2007) Survey of high-speed rough wall boundary layers: Invited presentation. In: 37th AIAA Fluid Dynamics Conference and Exhibit, American Institute of Aeronautics and Astronautics, Fluid Dynamics and Co-located Conferences

Discetti S, Ziskin IB, Astarita T, Adrian RJ, Prestridge KP (2013) Piv measurements of anisotropy and inhomogeneity in decaying fractal generated turbulence. Fluid Dyn Res 45(6):061401

Duan L, Martin M (2011) Direct numerical simulation of hypersonic turbulent boundary layers. part 4. effect of high enthalpy. J Fluid Mech 684:25-59

Duan L, Beekman I, Martin M (2010) Direct numerical simulation of hypersonic turbulent boundary layers. part 2. effect of wall temperature. J Fluid Mech 655:419-445

Duan L, Beekman I, Martin M (2011) Direct numerical simulation of hypersonic turbulent boundary layers. part 3. effect of mach number. J Fluid Mech 672:245-267

Dussauge JP, Smits AJ (1997) Characteristic scales for energetic eddies in turbulent supersonic boundary layers. Exp Thermal Fluid Sci 14(1):85-91

Ekoto I, Bowersox R, Beutner T, Goss L (2007) Response of a supersonic turbulent boundary layer to periodic surface roughness. In: 45th AIAA Aerospace Sciences Meeting and Exhibit

Ekoto IW, Bowersox R, Beutner T, Goss L (2008) Supersonic boundary layers with periodic surface roughness. AIAA J 46(2):486-497

Fenter FW (1960) The turbulent boundary layer on uniformly rough surfaces at supersonic speeds. University of Texas, Defense Research Laboratory

Fernholz HH, Finley P (1980) A critical commentary on mean flow data for two-dimensional compressible turbulent boundary layers. Tech. rep, DTIC Document

Grosser WI (1996) Factors influencing pitot probe centerline displacement error in a supersonic turbulent boundary layer. $\mathrm{PhD}$ thesis, Cleveland State University

Häberle J (2009) Untersuchungen zum externen und internen strömungsfeld eines scramjet triebwerkseinlaufs bei unterschiedlichen betriebspunkten. PhD thesis, Universität Stuttgart

Hannemann V (2002) Structured multigrid agglomeration on a data structure for unstructured meshes. Int J Numer Meth Fluids 40(3-4):361-368

Host-Madsen A, McCluskey DR (1994) On the accuracy and reliability of piv measurements. In: Seventh International Symposium on the Application of Laser Techniques to Fluid Mechanics, pp 1-11

Humble R, Scarano F, Van Oudheusden B (2007) Particle image velocimetry measurements of a shock wave/turbulent boundary layer interaction. Exp Fluids 43(2-3):173-183

Klebanoff P (1955) Characteristics of turbulence in boundary layer with zero pressure gradient. Tech. Rep. NACA-TR-1247, National Bureau of Standards
Klein M, Sadiki A, Janicka J (2003) A digital filter based generation of inflow data for spatially developing direct numerical or large eddy simulations. J Comput Phys 186(2):652-665

Latin RM (1998) The influence of surface roughness on supersonic high reynolds number turbulent boundary layer flow. Tech. rep, DTIC Document

Lee J, Monty J, Hutchins N et al (2016) Validating under-resolved turbulence intensities for piv experiments in canonical wall-bounded turbulence. Exp Fluids 57(8):129

Li X, Fu D, Ma Y (2008) Dns of compressible turbulent boundary layer around a sharp cone. Sci China, Ser G 51(6):699

Morkovin MV (1962) Effects of compressibility on turbulent flows. Mécanique de la Turbulence 367:380

Neeb D, Saile D, Gülhan A (2015) Experimental flow characterization and heat flux augmentation analysis of a hypersonic turbulent boundary layer along a rough surface. In: Proceedings of the 8th European Symposium on Aerothermodynamics for Space Vehicles, 89873, pp 1-15

Patel A, Boersma BJ, Pecnik R (2016) The influence of near-wall density and viscosity gradients on turbulence in channel flows. J Fluid Mech 809:793-820

Peltier S, Humble R, Bowersox R (2016) Crosshatch roughness distortions on a hypersonic turbulent boundary layer. Phys Fluids 28(4):045105

Peltier SJ (2013) Behavior of turbulent structures within a mach 5 mechanically distorted boundary layer. $\mathrm{PhD}$ thesis, Texas A\&M University

Priebe S, Martin M (2011) Direct numerical simulation of a hypersonic turbulent boundary layer on a large domain. In: $41 \mathrm{st}$ AIAA Fluid Dynamics Conference and Exhibit

Purtell L, Klebanoff P, Buckley F (1981) Turbulent boundary layer at low reynolds number. Phys Fluids 24(5):802-811

Ragni D, Schrijer F, Van Oudheusden B, Scarano F (2011) Particle tracer response across shocks measured by piv. Exp Fluids 50(1):53-64

Rakowitz M (2002) Grid refinement study with a uhca wing-body configuration using richardson extrapolation and grid convergence index gci. New results in numerical and experimental fluid mechanics III. Springer, New York, pp 297-303

Ringuette MJ, Martin MP, Smits AJ, Wu M (2006) Characterization of the turbulence structure in supersonic boundary layers using dns data. AIAA paper 3539

Roache PJ (1994) Perspective: a method for uniform reporting of grid refinement studies. Trans Am Soc Mech Eng J Fluids Eng 116:405-405

Sahoo D, Schultze M, Smits A (2009) Effects of roughness on a turbulent bloundary layer in hypersonic flow. In: 39th AIAA Fluid Dynamics Conference

Samimy M, Lele S (1991) Motion of particles with inertia in a compressible free shear layer. Phys Fluids A 3(8):1915-1923

Sillero JA, Jiménez J, Moser RD (2013) One-point statistics for turbulent wall-bounded flows at reynolds numbers up to $\delta+2000$. Phys Fluids 25(10):105102

Slater JW, Dudek JC, Tatum KE (2000) The nparc alliance verification and validation archive. In: Proceedings of ASME FEDSM00

So R, Zhang H, Gatski T, Speziale C (1994) Logarithmic laws from compressible turbulent boundary layers. AIAA J 32(11):2162-2168

Spencer A, Hollis D (2005) Correcting for sub-grid filtering effects in particle image velocimetry data. Meas Sci Technol 16(11):2323

Staff AR (1953) Equations, tables, and charts for compressible flow. NACA Report 1135

Tedeschi G, Gouin H, Elena M (1999) Motion of tracer particles in supersonic flows. Exp Fluids 26(4):288-296 
Thomas P (1991) Experimentelle und theoretische untersuchungen zum folgeverhalten von teilchen unter dem einfluß großer geschwindigkeitsgradienten in kompressibler strömung. $\mathrm{PhD}$ thesis, Universität Göttingen

Trettel A, Larsson J (2016) Mean velocity scaling for compressible wall turbulence with heat transfer. Phys Fluids 28(2):026102

Van Driest E (1952) Turbulent boundary layer on a cone in a supersonic flow at zero angle of attack. J Aeronaut Sci (Institute of the Aeronautical Sciences) 19(1):55-57

Van Driest ER (1951) Turbulent boundary layer in compressible fluids. J Aeronaut Sci 18:145-160

Van Driest ER (1956) On turbulent flow near a wall. J Aeronaut Sci 23:1007-1011

Wei T, Schmidt R, McMurtry P (2005) Comment on the clauser chart method for determining the friction velocity. Exp Fluids 38(5):695-699

White FM (2006) Viscous fluid flow, McGraw-Hill series in mechanical engineering, 3rd edn. McGraw-Hill Higher Education, New York and London
Widhalm M, Ronzheimer A, Meyer J (2008) Lagrangian particle tracking on large unstructured three-dimensional meshes. In: 46th AIAA Aerospace Sciences Meeting and Exhibit

Willems S, Gülhan A (2013) Experiments on shock induced laminarturbulent transition on a flat plate at mach 6. In: 5th European Conference for Aeronautics and Space Sciences (EUCASS), 1.-5. Jul. 2013, M++nchen, Deutschland

Williams OJ (2014) Density effects on turbulent boundary layer structure: from the atmosphere to hypersonic flow. $\mathrm{PhD}$ thesis, Princeton University

Williams OJH, Smits AJ (2017) Effect of tripping on hypersonic turbulent boundary-layer statistics. AIAA J 1-8

Williams OJ, Nguyen T, Schreyer AM, Smits AJ (2015) Particle response analysis for particle image velocimetry in supersonic flows. Phys Fluids 27(7):076101

Williams OJ, Sahoo D, Baumgartner ML, Smits AJ (2018) Experiments on the structure and scaling of hypersonic turbulent boundary layers. J Fluid Mech 834:237-270 